\title{
Equinoideos irregulares (Orden Spatangoida) del Cretácico de México
}

\author{
Alejandra Martínez Meloํㅡ, Blanca Estela Buitrón Sánchez ${ }^{1}$, Francisco Alonso Solís Marín ${ }^{2}$ \\ $\&$ Alfredo Laguarda Figueras ${ }^{2}$ \\ 1. Departamento de Paleontología, Instituto de Geología, Universidad Nacional Autónoma de México, Ciudad \\ Universitaria, CP.04510, Ciudad de México, México. \\ 2. Laboratorio de Sistemática y Ecología de Equinodermos, Instituto de Ciencias del Mar y Limnología, Universidad \\ Nacional Autónoma de México, Ciudad Universitaria, C.P. 04510, Ciudad de México, México. \\ Correspondencia: a.mtz.melo@gmail.com
}

Recibido 29-VII-2016. Corregido 06-IV-2017. Aceptado 23-VI-2017.

\begin{abstract}
Irregular echinoids (Order Spatangoida) from the Cretaceous of Mexico. Echinoids are a very important part of the Mesozoic and Cenozoic fossil fauna of Mexico. Among them, spatangoids are widely represented in several Cretaceous fossiliferous outcrops, and have stratigraphical and paleoecological value due to their good preservation of the Cretaceous. The review of order Spatangoida L. Agassiz, 1840 from the Cretaceous in Mexico allowed un update the taxonomy of species and contributed to the study of paleogeographic and stratigraphic distribution. The order Spatangoida is the most diverse in Mexico with three families (Toxasteridae, Hemiasteridae, Micrasteridae), seven genera (Epiaster, Heteraster, Macraster, Washitaster, Hemiaster, Proraster, Micraster) and 21 species. Family Toxasteridae is the most numerous with $54 \%$ of the species reported for the Cretaceous of Mexico. Spatangoids from the Cretaceous of Mexico are found in different localities in Sonora (Cerro de las Conchas), Chihuahua (Cerro de Muleros, Sierra de Juárez, Placer de Guadalupe, Sierras La Ranchera, El Presidio, Sierra Banco de Lucero, Sierra La Encantada, Villa Ahumada), Coahuila (Sierra de Tlahualilo), Nuevo León (Sinclinal de Delgado), San Luis Potosí (Zamachihue), Colima (El Rosario), Puebla (San Juan Raya, Barranca Salitrillo), Guerrero (Cerro de Ahuexotitlán) and Oaxaca (Las Huertas). Rev. Biol. Trop. 65(Suppl. 1): S113-S136. Epub 2017 November 01.
\end{abstract}

Key words: Irregular echinoids; Spatangoida; Cretaceous; Mexico.

El Cretácico es un periodo especial en la historia de la Tierra; marcó uno de los periodos más activos en la evolución y la diversificación de la biota, particularmente de los equinodermos, que fueron fuertemente afectados por cambios ambientales rápidos $\mathrm{y}$ a gran escala. Durante este periodo se desarrollaron ampliamente las paleocomunidades marinas bentónicas, comparables estructural y taxonómicamente con las comunidades modernas. Hace aproximadamente $100 \mathrm{Ma}$ ocurrió una transgresión y regresión global simultánea, que sumergió aproximadamente un tercio de la superficie continental actual del territorio mexicano (Prothero \& Dott, 2004).
Los equinoideos cretácicos procedentes de diversas localidades de 17 estados de México han sido motivo de numerosas publicaciones, entre ellas Cotteau (1890), quien propuso una nueva especie (Heteraster mexicanus) para México. Böse (1910) estudió los equinoideos del Cerro de Muleros, Chihuahua, y Böse y Cavins (1927) los del sur de E.U.A. y norte de México. Buitrón (1968) hizo el catálogo de equinoideos fósiles mexicanos; más tarde, Buitrón (1970) estudió los equinoideos del Cretácico de Puebla y en 1971 publicó la paleontología sistemática de 22 especies de equinoideos de los estados de Sonora, Chihuahua y Coahuila y propuso una especie nueva, 
Micraster (Gibbaster) sonorensis. Buitrón (1976) describió los equinoideos de Guerrero y la misma autora en 1978 correlacionó la fauna de equinoideos de dichas regiones para el Cretácico Tardío.

Buitrón (1990) y Buitrón y Solís-Marín (1993) publicaron el registro de los equinoideos del Jurásico y Cretácico de México, y su distribución geográfica y estratigráfica en el país. Nieto y García (2006) realizaron un listado con 65 especies procedentes de 17 estados del territorio mexicano, entre ellas 20 de equinoideos regulares y 45 de equinoideos irregulares.

En México los espatangoides cretácicos se encuentran distribuidos en los estados de Sonora (Cerro de las Conchas), Chihuahua (Cerro de Muleros, Sierra de Juárez, Placer de Guadalupe, Sierras La Ranchera, El Presidio, Sierra Banco de Lucero, Sierra La Encantada, Villa Ahumada), Coahuila (Sierra de Tlahualilo), Nuevo León (Cuenca la Popa, Sinclinal de Delgado), San Luis Potosí (Zamachihue), Colima (El Rosario), Puebla (San Juan Raya, Barranca Salitrillo), Guerrero (Cerro de Ahuexotitlán) y Oaxaca (Las Huertas) (Fig. 1).
Aunque el conocimiento sobre los equinoideos cretácicos en México se ha generado desde hace más de 100 años, han sido pocos los esfuerzos para reunir la información taxonómica de las especies reportadas. El trabajo más reciente (Nieto \& García, 2006), que sirvió como base para éste, enlista las especies de equinoideos del Cretácico en México; en él se ofrece información sobre el primer reporte de las especies y los estados de la República en los que se han registrado.

Lamentablemente dicho trabajo presenta problemas evidentes; se mencionan algunas especies albergadas en la Colección Paleontológica Nacional (Instituto de Geología, UNAM), sin embargo, dichos ejemplares no existen dentro de la colección ni en su base de datos; se incluyen especies del orden Clypeasteroida, cuando su origen está dado para el Paleoceno (Smith, 1984), por último, no se permite relacionar las especies con las localidades donde han sido reportadas.

Es por ello que, en el presente trabajo, se hace una revisión de la información taxonómica de los equinoideos irregulares reportados

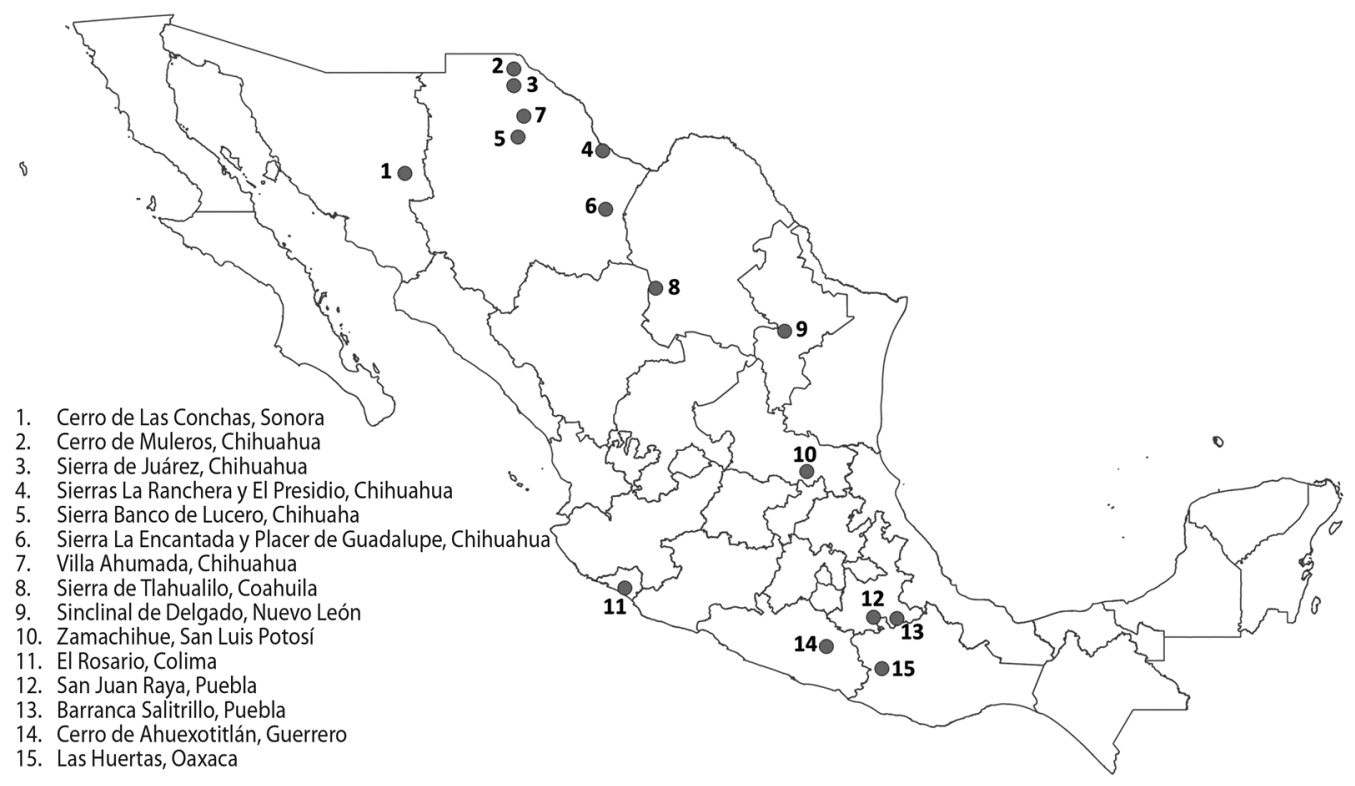

Fig. 1. Mapa con registros del Orden Spatangoida durante el Cretácico en México.

Fig. 1. Map with records of Spatangoida during the Cretaceous in Mexico. 
para el Cretácico en México, haciendo una búsqueda de ejemplares en colecciones nacionales e internacionales o, en su defecto, de reportes formales. Esta revisión, junto con el trabajo de Nieto y García (2006), sientan las bases para el conocimiento real de de la diversidad taxonómica y morfológica del grupo en el país.

\section{MATERIAL Y MÉTODOS}

La presente investigación se basó en la lista de los equinoideos reportados para el Cretácico de México, publicada por Nieto y García (2006), de la cual se revisó la validez taxonómica. Se complementó la lista con el estudio de nuevos registros de la Colección Nacional de Paleontología del Instituto de Geología (IGM) y del Museo de Paleontología de la Facultad de Ciencias de la Universidad Nacional Autónoma de México (FCMP); dichos ejemplares se determinaron y clasificaron bajo el criterio de Kroh y Smith (2010).

Por último, se eliminaron todos los datos que no se pudieron corroborar con reportes bibliográficos formales o en colecciones paleontológicas.

De la lista de especies resultantes se obtuvo información de sinonimias, material tipo, descripción, distribución y se menciona el material examinado. Muchos reportes son muy antiguos y no ofrecen toda la información que se maneja actualmente, por lo que en algunos casos se menciona la presencia de las especies, pero no existen datos morfológicos o de la serie tipo, es por ello que los datos de las especies se complementan con una lámina ilustrando las vistas aboral, oral, lateral y posterior de ejemplares de las especies. Algunas especies solo cuentan con ejemplares albergados en la Springer Collection, USNM y, aunque no cuentan con información de recolecta, su calidad de preservación y la validez de identificación permiten usarlos para ilustrar las especies.

\section{RESULTADOS}

\section{Paleontología sistemática}

\section{ORDEN SPATANGOIDA L. Agassiz, 1840}

Diagnosis. Testa cordiforme o circular con hendidura en el margen anterior, sin bourrelets. Ambulacros pares ligeramente petaloides; en el área oral el ambulacro posterior forma un plastrón. Floscele no diferenciado. Filodios conspicuos. Sistema apical posterior y peristoma anterior, sin hendiduras branquiales. Periprocto posterior o inframarginal. Fasciolas con desarrollo variable, a veces ausentes particularmente en formas primitivas (Fischer, 1966).

\section{FAMILIA Toxasteridae Lambert, 1920}

Diagnosis. Ambulacros pares, petaloides abiertos. Ambulacro impar petaloide o no. Sistema apical etmofracto. Plastrón protanfisterno o mesanfisterno. Generalmente sin fasciolas y $\sin$ espinas primarias. Cretácico InferiorReciente (Fischer, 1966; Akers \& Akers, 1987).

Género Epiaster d’Orbigny, 1853

Diagnosis. Testa cordiforme, con surco anterior ligeramente hundido, región posterior trunca, superficie oral plana. Ambulacros pares petaloides abiertos, con poros alargados. Ambulacro anterior ligeramente hundido. Tubérculos aborales pequeños y uniformes, rodeados de gránulos finos. Sistema apical etmofracto con cuatro gonoporos centrales. Periprocto pequeño posterior. Peristoma pequeño anterior. Sin fasciolas (Smith \& Kroh, 2011).

Especie tipo. Epiaster trigonalis (Desor, 1847).

Alcance estratigráfico. Cretácico Inferior (Barremiano-Albiano). 
Epiaster dartoni Cooke, 1955

Figura 2

Epiaster dartoni Cooke, 1955,

p. 108, lám. 28, figs. 1-10.

Macraster dartoni Buitrón, 1971,

p. 31, lám. 8, figs. 2, 3; lám. 9, figs. 2, 3, 5, 6 .

Material tipo. Smithsonian Museum of Natual History, Sintipos USNM PAL 328459, USNM MO 108711 y 108722.

Descripción. Testa cordiforme, truncada en la región posterior. Ambulacro anterior hundido, poros pequeños en forma de "V". Ambulacros pares moderadamente deprimidos, el par anterior ligeramente más largo que el posterior con poros alargados transversalmente. Sistema apical subcentral, cuatro placas genitales contiguas con gonoporo. Placa madrepórica central y dos de las placas oculares posteriores son contiguas. Peristoma pequeño, subpentagonal, anterior. Periprocto alargado y puntiagudo en los extremos. Tubérculos escasos y más concentrados en el plastrón (Cooke, 1955).

Distribución. Aptiano, Grant County, New Mexico, E.U.A. Arroyo Palomas, cerca del Rancho San Vicente, Coahuila; Albiano, en Formación Benigno, Sierra de Presidio y Formación Lágrima, Sierra de la Ranchera, Chihuahua (Buitrón, 1971).

Material examinado: IGM 2262 y 2263.

Género Heteraster d'Orbigny, 1853

Diagnosis. Testa cordiforme, ambulacro impar semipetaloide, con poros que alternan con ranuras pequeñas y alargadas; en algunas especies la zona porífera anterior de los ambulacros pares es mucho más estrecha que las zonas posteriores (modificada de Fischer, 1966).

Especie tipo. Heteraster oblongus (Brongniart, 1821).

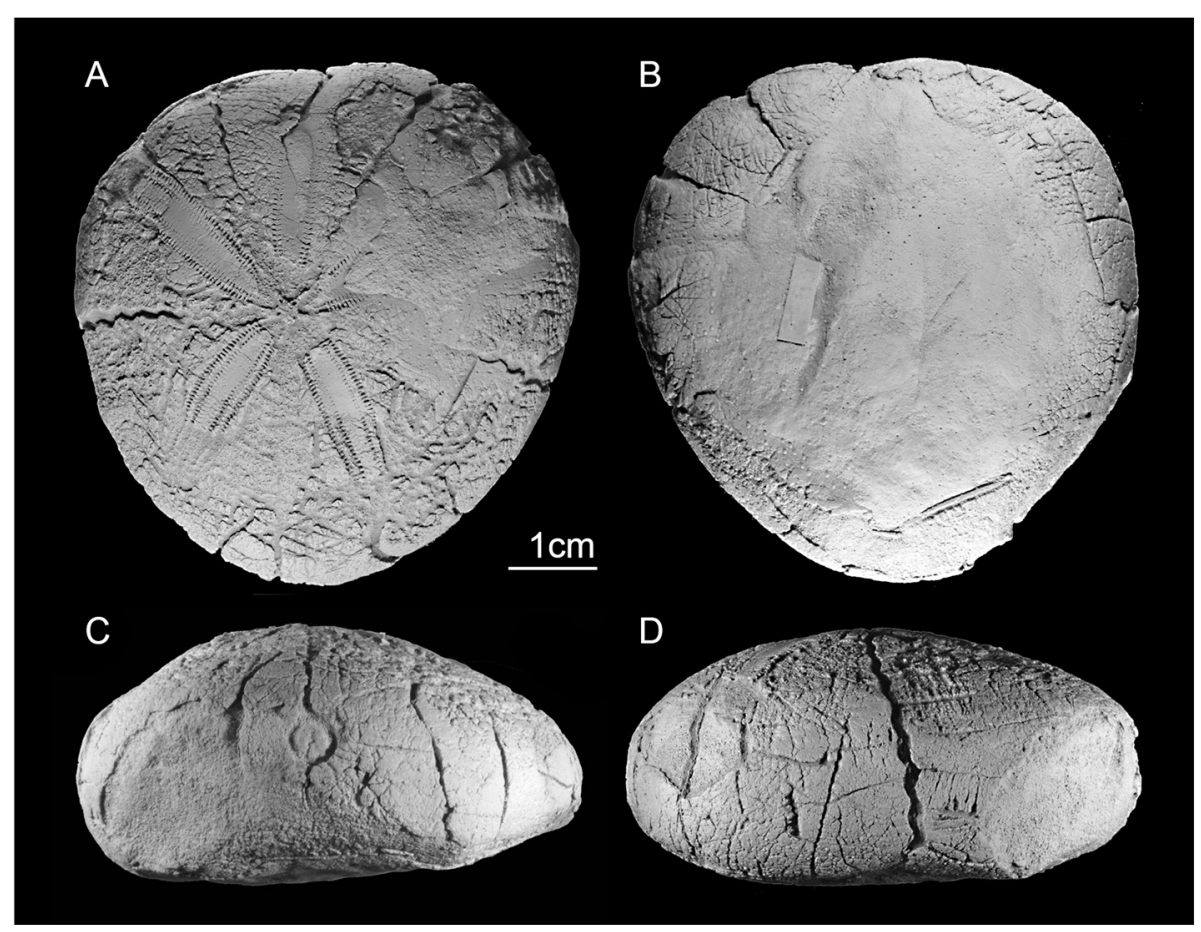

Fig. 2. Epiaster dartoni Cooke, 1955. IGM 2262 (Formación Lágrima, Albiano). A, vista aboral; B, vista oral; C, vista posterior; D, vista lateral.

Fig. 2. Epiaster dartoni Cooke, 1955. IGM 2262 (Lagrima Formation, Albian). A, aboral view; B, oral view; C, posterior view; D, lateral view. 
Alcance estratigráfico. Cretácico (Barremiano-Cenomaniano).

Heteraster aguilerai Buitrón, 1970

Figura 3

Heteraster aguilerai Buitrón, 1970, p. 32-34, lám. 7, figs. 1-5.

Material tipo. Holotipo IGM 2227, Formación San Juan Raya, Aptiano.

Descripción. Testa pequeña cordiforme, muy alta, superficie aboral convexa con el sistema apical ligeramente anterior; superficie oral plana, exceptuando la pequeña hendidura donde se localiza el peristoma. Región anterior más ancha que la posterior, con un surco poco profundo. Región posterior truncada. Ambulacros pares subpetaloides, los anteriores más largos y curvos, ambulacros posteriores cortos con poros en forma de ranura pequeña de igual tamaño. Ambulacro anterior impar en un surco. Sistema apical pequeño y compacto, con cuatro placas genitales grandes y cinco placas oculares pequeñas, la madreporita es central. Periprocto posterior sobre la superficie aboral de la testa. Peristoma ligeramente ovalado y en posición anterior (modificada de Buitrón, 1970).

Distribución. Barranca Salitrillo, San Juan Raya, Puebla (Buitrón, 1970).

Material examinado: IGM 2227, Formación San Juan Raya, Aptiano.

\section{Heteraster alencasterae Buitrón, 1970}

Figura 4

Heteraster alencasterae Buitrón, 1970, p. 34-35, lám. 6, figs. 1-6.

Material tipo. Holotipo IGM 2228 y Paratipo IGM 2229, Formación San Juan Raya, Aptiano.

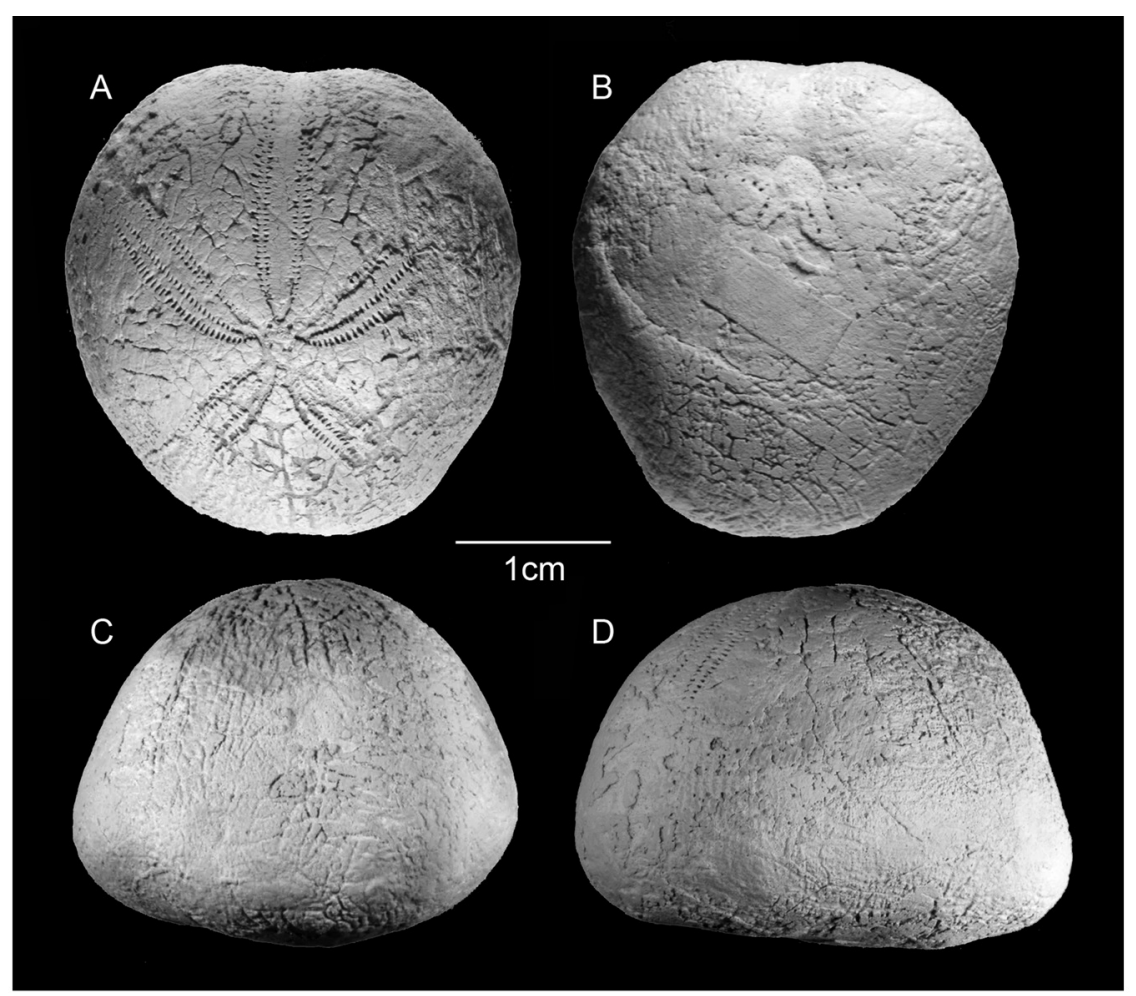

Fig. 3. Heteraster aguilerai Buitrón, 1970. Holotipo IGM 2227 (Formación San Juan Raya, Aptiano). A, vista aboral; B, vista oral; C, vista posterior; D, vista lateral.

Fig. 3. Heteraster aguilerai Buitrón, 1970. Holotype IGM 2227 (San Juan Raya Formation, Aptian). A, aboral view; B, oral view; C, posterior view; D, lateral view. 


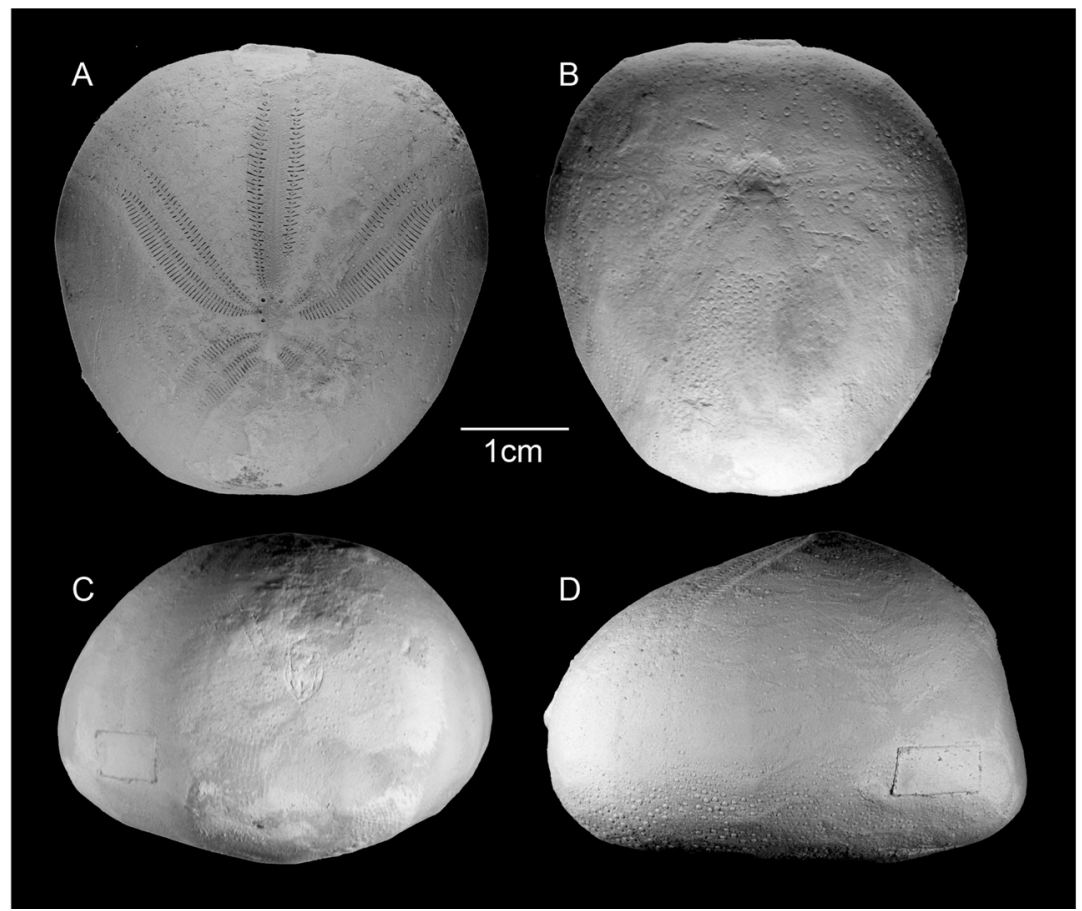

Fig 4. Heteraster alencasterae Buitrón, 1970. Holotipo IGM 2228 (Formación San Juan Raya, Aptiano). A, vista aboral; B, vista oral; C, vista posterior; D, vista lateral.

Fig. 4. Heteraster alencasterae Buitrón, 1970. Holotype IGM 2228 (San Juan Raya Formation, Aptian). A, aboral view; B, oral view; C, posterior view; D, lateral view.

Descripción. Testa cordiforme, grande, con el ápice en la región posterior. Superficie aboral convexa con un surco estrecho en la región anterior, sin dividir el margen anterior. Extremo posterior trunco, de perfil oblicuo. Superficie oral plana, ligeramente cóncava hacia el peristoma. Ambulacros subpetaloides, los anteriores más largos que los posteriores; ambulacro anterior impar hundido. Poros alargados y dispuestos en pares de diferente tamaño que alternan de manera irregular. Placas interambulacrales grandes, con tubérculos grandes areolados, crenulados y perforados, rodeados por numerosos gránulos que ocupan el resto de la placa. Sistema apical pequeño y compacto, situado en el ápice de la testa, con cuatro placas genitales grandes, cinco placas oculares y la madreporita en posición central (Buitrón, 1970).

Distribución. Barranca Salitrillo, San Juan Raya, Puebla (Buitrón, 1970).
Material examinado: Holotipo IGM 2228 y Paratipo IGM 2229, Formación San Juan Raya, Aptiano.

Heteraster mexicanus (Cotteau, 1890)

Figura 5

Enallaster mexicanus Cotteau, 1890, p. 296, lám. 2, figs. 1-8. Cooke, 1946, p. 231. Cooke, 1955, p. 103, lám. 26, figs. 1-8. Akers \& Akers, 1987, p. 88.

Heteraster mexicanus. Adkins, 1928, p. 292, lám.8, fig. 2. Buitrón, 1971, p. 28, lám.7, figs. 4-8. Akers y Akers, 1987, p. 88.

Material tipo. No disponible.

Descripción. Testa ovalada a cordiforme, de ámbito redondeado y con extremo posterior trunco. Superficie aboral convexa, con el vértice en el sistema apical, que es ligeramente posterior; con un surco profundo en el ambulacro anterior. Ambulacro impar ancho 


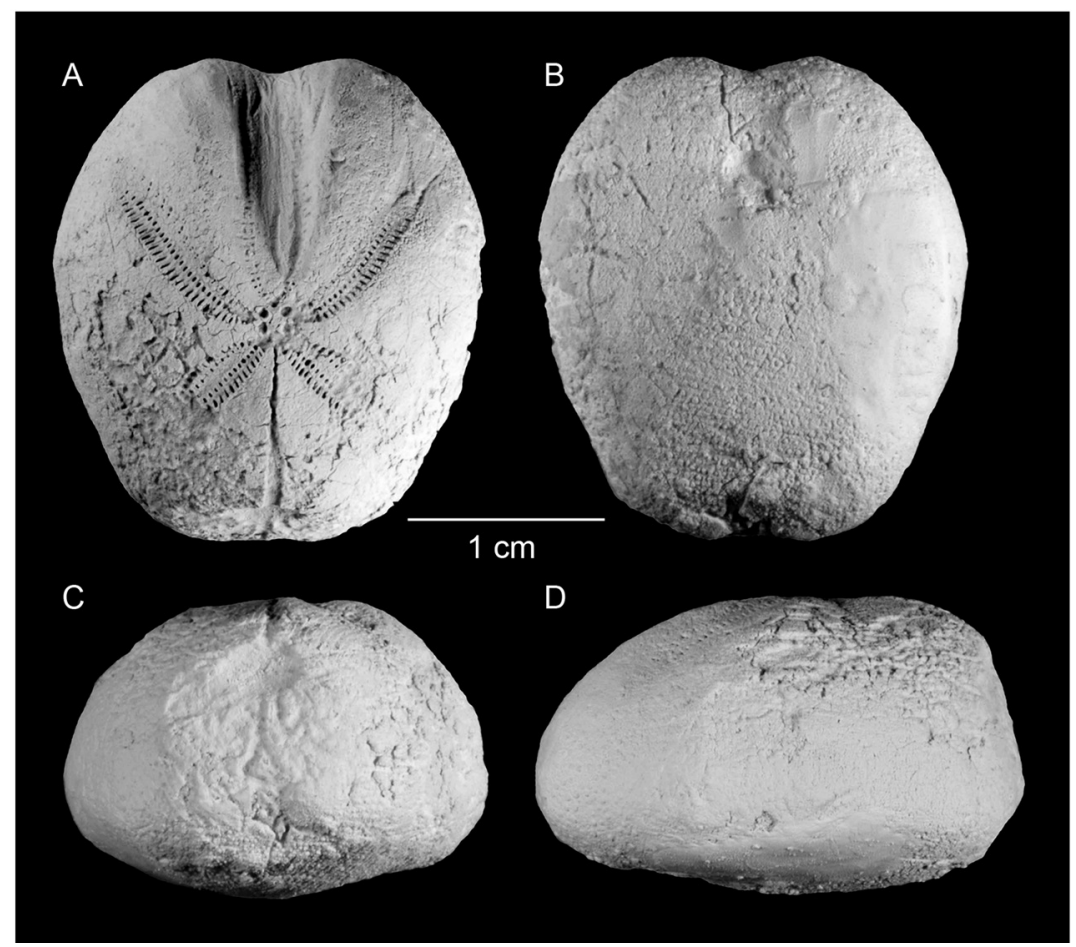

Fig 5. Heteraster mexicanus (Cotteau, 1890). FCMP 81 (Albiano). A, vista aboral; B, vista oral; $\mathrm{C}$, vista posterior; D, vista lateral.

Fig. 5. Heteraster mexicanus (Cotteau, 1890). FCMP 81 (Albian). A, aboral view; B, oral view; $\mathrm{C}$, posterior view; $\mathrm{D}$, lateral view.

y semipetaloide con placas provistas de un poro y una hendidura grande que alternan de manera regular con otras de un poro y una hendidura pequeña. Ambulacros anterolaterales más largos que los posteriores y flexionados ligeramente hacia la parte posterior; con pares de poros pequeños, ovalados horizontalmente. Ambulacros posteriores pequeños y divergentes, casi rectos, con zona interporífera amplia; presenta pares de ranuras, las internas pequeñas y las externas grandes, en forma de coma. Ambulacro anterior estrecho, constituido por pares de poros redondos, del mismo tamaño. Sistema apical localizado en la parte posterior de la testa; con cuatro placas genitales grandes, pentagonales y perforadas, las posteriores se tocan en la base; madreporita central; cinco placas oculares pequeñas y triangulares, las posteriores contiguas. Periprocto pequeño, ovalado transversalmente y situado en la parte superior de la pared posterior. Peristoma pequeño, casi pentagonal, localizado en una pequeña depresión, en la parte anterior de la base de la testa; plastrón abultado (Buitrón, 1971).

Distribución. Cretácico Temprano; Formaciones Comanche Peak, Edwards, Goodland y Walnut Clay, Texas, E. U. A. Albiano, Formación Mal Paso. Albiano medio en La Encantada, cerca del Placer de Guadalupe, Chihuahua (Buitrón, 1971).

Material examinado: FCMP 75-82. Albiano, Formación Mal Paso.

Heteraster obliquatus (W.B. Clark, 1893)

Figura 6

Enallaster obliquatus W.B. Clark, 1893, p. 79. Cooke, 1955, p. 102.

Enallaster texanus (Roemer). Cooke, 1946, p. 231.

Heteraster obliquatus. Akers \& Akers, 1987, p. 88. Finsley, 1999, p. 108. 


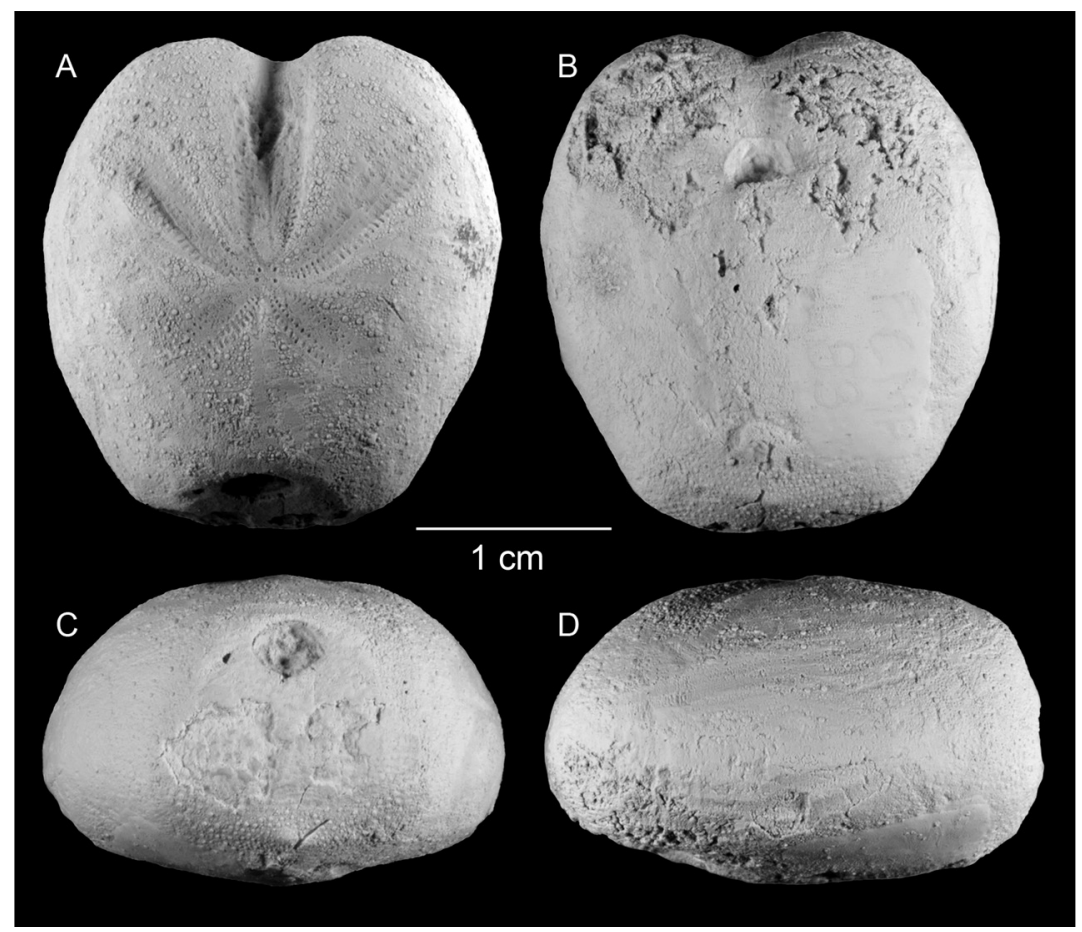

Fig 6. Heteraster obliquatus (W.B. Clark, 1893). FCMP 83 (Albiano). A, vista aboral; B, vista oral; $\mathrm{C}$, vista posterior; $\mathrm{D}$, vista lateral.

Fig. 6. Heteraster obliquatus (W.B. Clark, 1893). FCMP 83 (Albian). A, aboral view; B, oral view; $\mathrm{C}$, posterior view; D, lateral view.

Material tipo. No disponible.

Descripción. Testa cordiforme con el extremo posterior truncado. Superficie aboral convexa y elevada posteriormente; superficie oral plana con una elevación en el plastrón. Ambulacros estrechos y desiguales. Tubérculos pequeños. Sistema apical posterior. Periprocto pequeño; peristoma pequeño, redondo, muy anterior (modificada de Akers y Akers, 1987).

Distribución. Aptiano, Grant County, New Mexico; Formación, Glen Rose, Texas, E.U.A.

Material examinado: FCMP 83-84. Albiano.

Heteraster texanus Roemer, 1849

Figura 7

Heteraster texanus Roemer, 1849.

Akers \& Akers, 1987, p. 89.
Descripción. Testa de ámbito ovalado, más ancha en la parte anterior y trunca en el extremo posterior. Presenta un surco anterior donde está el ambulacro impar. Superficie aboral convexa y la oral plana con una depresión a la altura del peristoma, que es ovalado transversalmente. Ambulacros estrechos y desiguales en longitud; el par posterior más corto que el anterior. Sistema apical muy compacto. Periprocto en la parte alta posterior de la testa (Akers \& Akers, 1987).

Distribución. Cretácico; Texas, E.U.A.

Material examinado: Springer Collection, USNM.

Heteraster trauthi (Kühn, 1925)

Epiaster trauthi Kühn, 1925, p. 186-187.

Heteraster trauthi. Sánchez-Rodríguez, 1997, p. 34. Nieto \& García, 2006, p. 107.

Material tipo. No disponible.

Material tipo. No disponible. 


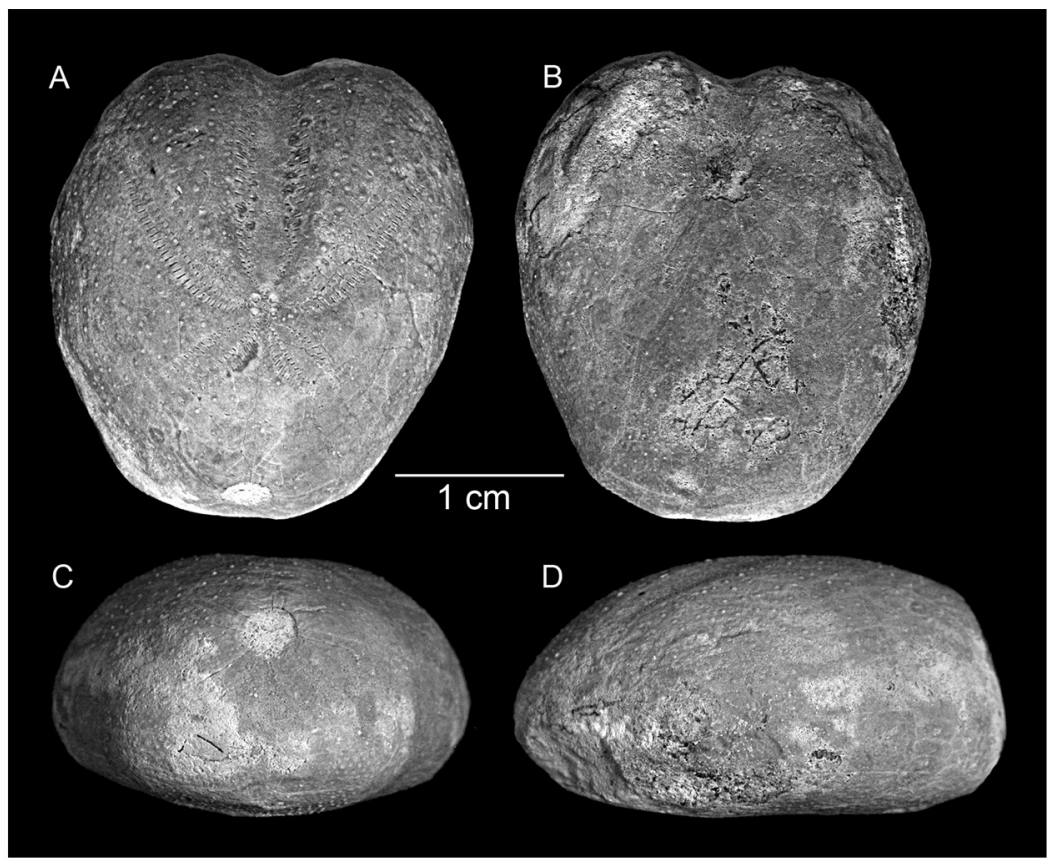

Fig. 7. Heteraster texanus Roemer, 1849. Springer Collection, USNM. A, vista aboral; B, vista oral; $\mathrm{C}$, vista posterior; $\mathrm{D}$, vista lateral.

Fig. 7. Heteraster texanus Roemer, 1849. Springer Collection, USNM. A, aboral view; B, oral view; $\mathrm{C}$, posterior view; D, lateral view.

Diagnosis. Testa con ámbito redondo, borde posterior trunco y más elevado que el anterior. Superficie aboral con pendiente hacia el margen anterior, cubierta por pequeños tubérculos perforados. Superficie oral plana, con excepción del labrum, que es prominente. Ambulacro impar recto y más ancho que los pares, con poros pequeños, redondos; cada poro está separado por un nódulo conspicuo. Ambulacros anteriores rectos y más largos que los posteriores, que son ligeramente flexionados; con poros transversalmente alargados. Fasciola periambulacral angosta. Sistema apical tetrabasal, con cuatro placas genitales perforadas. Peristoma hundido, más alto que ancho (Sánchez-Rodríguez, 1997).

Distribución. Formación Cárdenas, Zamachihue, San Luis Potosí (Sánchez-Rodríguez, 1997). Cretácico (Maastrichtiano).

Material examinado: Reporte en Sánchez-Rodríguez, 1997.
Heteraster wenoensis (Adkins, 1920)

Figura 8

Enallaster wenoensis Adkins, 1920, p. 112, lám. 5, fig. 3 .

Heteraster (Enallaster) wenoensis.

Lambert, 1927, p. 64, 271.

Washitaster wenonensis. Akers \& Akers, 1987, p. 111.

Material tipo. No disponible.

Descripción. Testa con contorno ovalado y ensanchada anteriormente. Ambulacro impar con pares de poros separados por un tubérculo. Ambulacros anteriores flexionados hacia el extremo anterior; ambulacros posteriores cortos. Sistema apical posterior. Periprocto ovalado y situado en la parte superior de la superficie posterior trunca. Peristoma ovalado (Akers \& Akers, 1987).

Distribución. Cretácico; Texas, E.U.A.

Material examinado: FCMP 85. Albiano. 


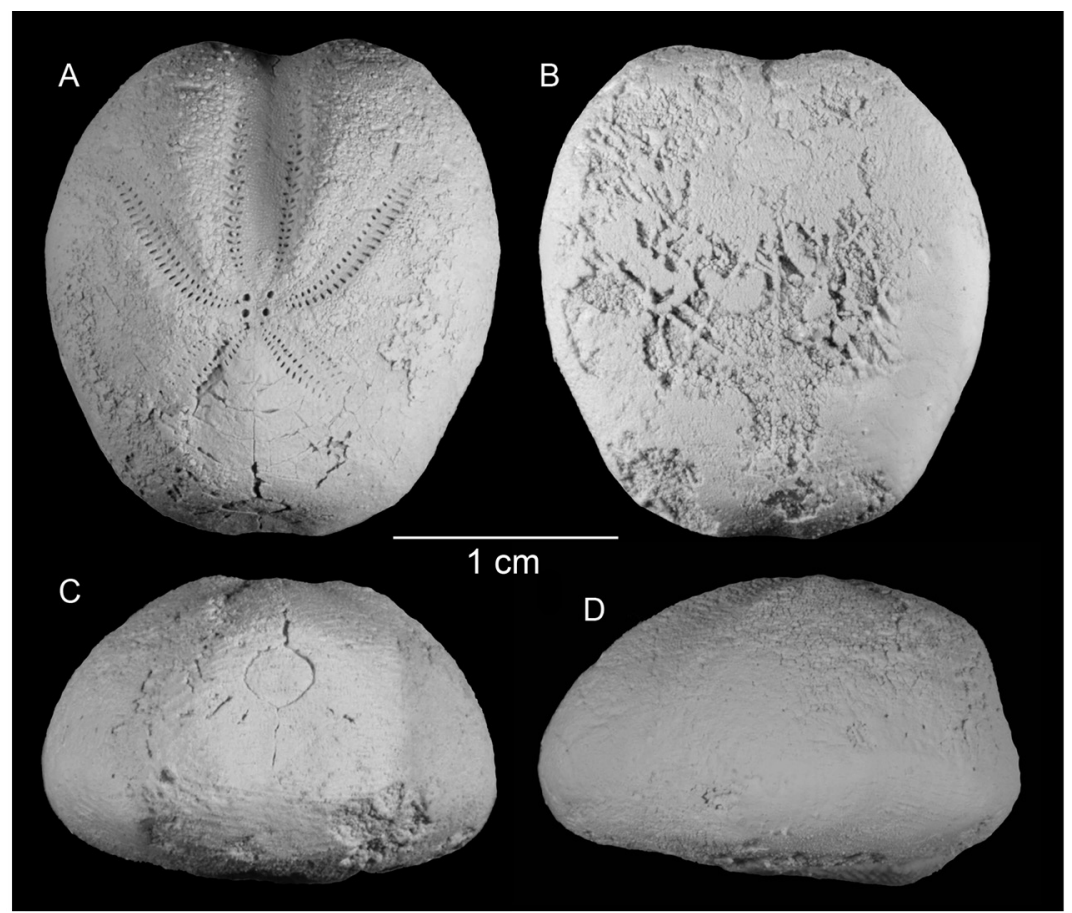

Fig. 8. Heteraster wenoensis (Adkins). FCMP 85 (Albiano). A, vista aboral; B, vista oral; $\mathrm{C}$, vista posterior; $\mathrm{D}$, vista lateral.

Fig. 8. Heteraster wenoensis (Adkins). FCMP 85 (Albian). A, aboral view; B, oral view; $\mathrm{C}$, posterior view; $\mathrm{D}$, lateral view.

Género Macraster Roemer, 1888

Diagnosis. Testa ovalada con ámbito redondeado, con surco anterior poco profundo; superficie oral plana y ligeramente deprimida; superficie posterior trunca. Ambulacros petaloides largos, paralelos y abiertos distalmente; ambulacro impar hundido desde el ápice hasta el peristoma. Parafasciola peripétala. Tubérculos aborales uniformes y pequeños. Sistema apical central, etmofracto con cuatro gonoporos. Periprocto pequeño central en la superficie posterior. Peristoma pequeño y ovalado, en posición anterior; placa del labio larga y triangular, con punta extendiéndose a tocar la placa 5a2; placas esternales grandes y asimétricas con sutura oblicua (Smith \& Kroh, 2011).

Especie tipo. Macraster texanus Roemer, 1888.

Alcance estratigráfico. Cretácico (Albiano al Cenomaniano) (Smith \& Kroh, 2011).
Macraster aguilerae (Böse, 1910)

Figura 9

Epiaster aguilerae Böse, 1910, p.173, lám. 47, figs. 2-4, 6-7, lám. 48, figs. 1-2, 4. Macraster aguilerae (Böse). Buitrón 1971 p. 30, lám. 8, figs. 1, 4, 5; lám 9, figs. 1, 4. Akers \& Akers, 1987, p. 94.

Material tipo. Sintipo IGM 2261, Albiano tardío.

Descripción. Testa grande, de ámbito cordiforme, con el ápice entre el sistema apical y el límite posterior; con surco escotando el ámbito anterior. Superficie aboral ligeramente convexa; superficie oral plana, con una ligera concavidad hacia el peristoma y ligeramente inflada en el plastrón. Ambulacro impar alojado en el surco, zonas poríferas amplias con poros iguales en forma de ranuras grandes. Ambulacros pares anteriores largos y casi rectos, con las zonas porífera e interporífera 


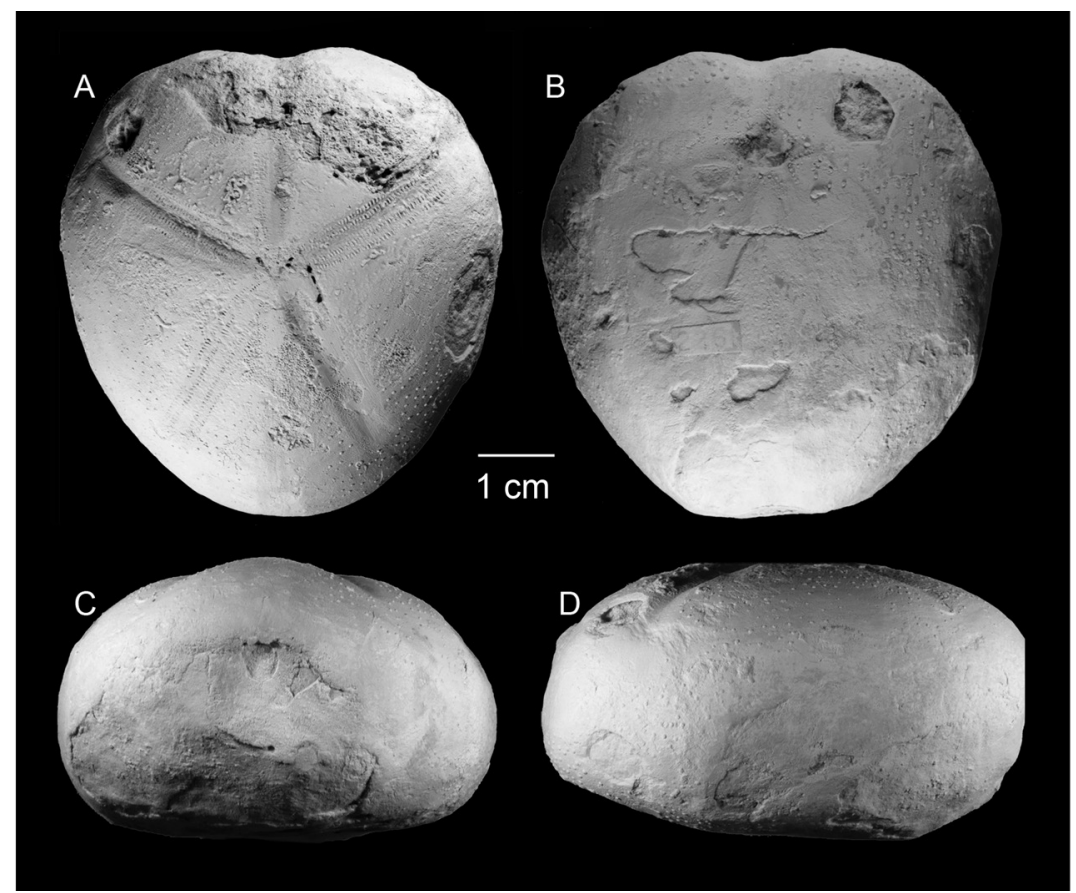

Fig. 9. Macraster aguilerae (Böse, 1910). Sintipo IGM 2261 (Cerro de Muleros, Albiano tardío). A, vista aboral; $\mathrm{B}$, vista oral; C, vista posterior; D, vista lateral.

Fig. 9. Macraster aguilerae (Böse, 1910). Syntype IGM 2261 (Cerro de Muleros, late Albian). A, aboral view; $\mathrm{B}$, oral view; $\mathrm{C}$, posterior view; $\mathrm{D}$, lateral view.

amplias con poros iguales en forma de ranura. Ambulacros posteriores rectos y cortos; la zona porífera con poros iguales, en forma de ranuras. Sistema apical pequeño y excéntrico hacia la parte anterior. Periprocto ovalado, situado en la parte superior de la pared posterior. Peristoma oval (modificada de Buitrón, 1971).

Distribución. Cretácico (Albiano superior) en Cerro de Muleros, Chihuahua (Buitrón, 1971).

Material examinado: Sintipo IGM 2261, Albiano tardío.

\section{Macraster texanus Roemer, 1888}

Figura 10

Macraster texanus Roemer, 1888, p. 191. Akers \& Akers, 1987, p. 101.

Material tipo. No disponible.

Descripción. Testa de ámbito ovalado y aplanada en ambas superficies, plastrón elevado posteriormente, ambulacros largos y rectos, ambulacro impar ligeramente hundido y con poros en forma de "V"; interambulacros ligeramente elevados. Fasciola peripétala tenue. Sistema apical ligeramente anterior, con cuatro poros genitales. Periprocto pequeño y circular, en la parte media de la superficie posterior. Peristoma anterior, subpentagonal y sin un labrum conspicuo (modificada de Akers \& Akers, 1987).

Distribución. Albiano; Formación Anacacho, Uvalde County, Texas, E.U.A.

Material examinado: Springer Collection, USNM.

Género Washitaster Lambert, 1927

Washitaster Lambert, 1927, p. 271.

Diagnosis. Testa ovalada con surco anterior distintivo; superficie oral oblicua trunca. Testa deprimida vista lateralmente. Sistema 


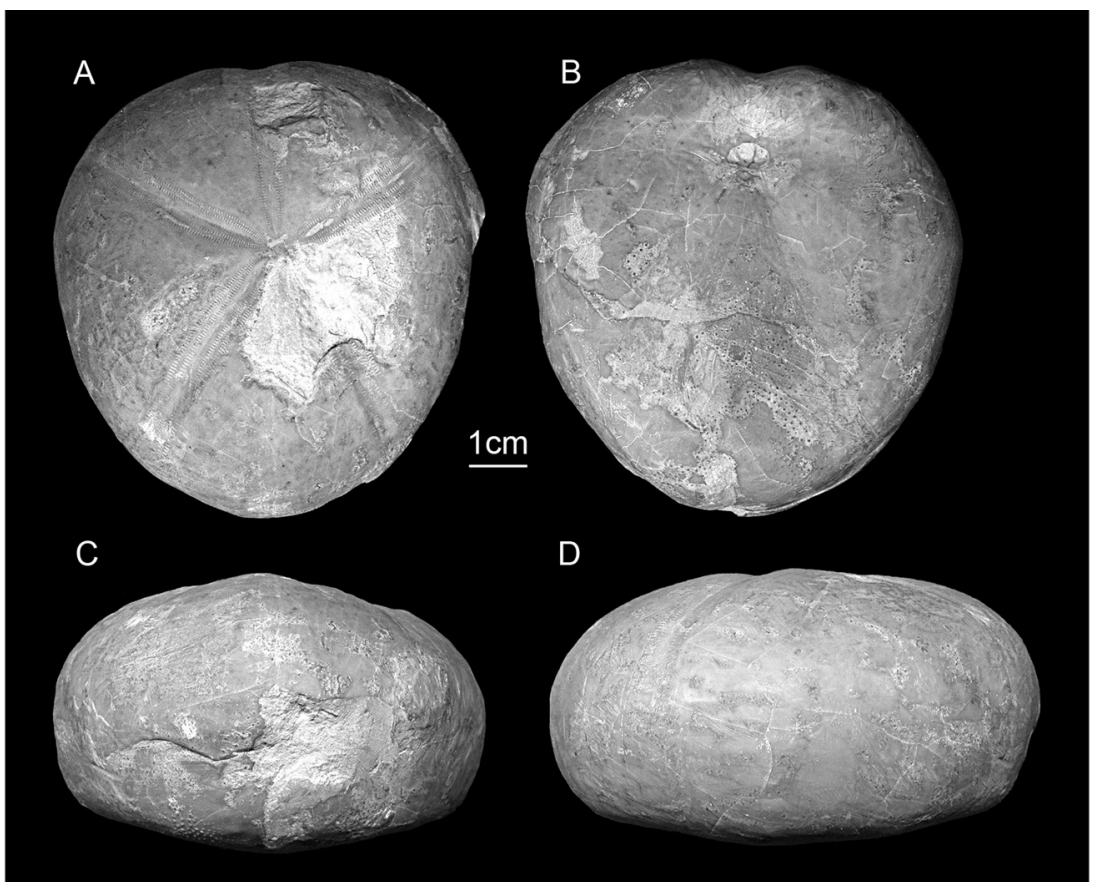

Fig. 10. Macraster texanus Roemer, 1888. Springer Collection, USNM. A, vista aboral; $\mathrm{B}$, vista oral; C, vista posterior; D, vista lateral.

Fig. 10. Macraster texanus Roemer, 1888. Springer Collection, USNM. A, aboral view; B, oral view; $\mathrm{C}$, posterior view; D, lateral view.

apical etmofracto con cuatro gonoporos, en posición ligeramente posterior. Ambulacro anterior hundido desde el ápice; la mayoría de los pares de poros son estrechos. Ambulacros anteriores petaloides largos y ligeramente curvos anteriormente, con la hilera anterior con pares de poros en la parte distal. Ambulacros posteriores petaloides más cortos. Peristoma cercano al borde anterior de la testa y con labrum alargado proyectándose hasta la segunda placa ambulacral. Placas esternales asimétricas con sutura oblicua. Periprocto posterior pequeño y redondo. Múltiples bandas de fasciolas rodean a los ambulacros. Tubérculos aborales distintivamente más largos hacia el ápice en los interambulacros anteriores (Smith \& Kroh, 2011).

Especie tipo: Hemiaster riovistae Adkins, 1920.

Alcance estratigráfico: Cretácico (Albiano) en E.U.A. y México (Smith \& Kroh, 2011).
Washitaster bravoensis (Böse, 1910)

Figura 11

Enallaster bravoensis Böse, 1910, p. 168, lám. 41, figs. 5-10; lám. 42, figs. 2-12, lám. 43, figs. 1-2, 6-7. Whitney, 1916, p. 100, lám. 20, fig. 3-5. Adkins \& Winton, 1920, p. 58, lám. 9, fig. 11 .

Enallaster (Washitaster) bravoensis. Cooke, 1955, p. 106-107, lám. 27, figs. 5-12. Washitaster bravoenisis. Buitrón, 1971, p. 37, lám.11, figs. 4-9. Buitrón, 1978, p. 69.

Buitrón, 1990, fig. 1-4.

Material tipo. Sintipos IGM 2274, 2275, 2276, 2277, 2278. Albiano tardío.

Descripción. Testa ovalada, con surco anterior en el ambulacro impar, ligeramente deprimida anteriormente y con el extremo posterior trunco y alto. Ambulacro anterior impar no petaloide, hundido en un surco que forma una escotadura en el borde anterior. 


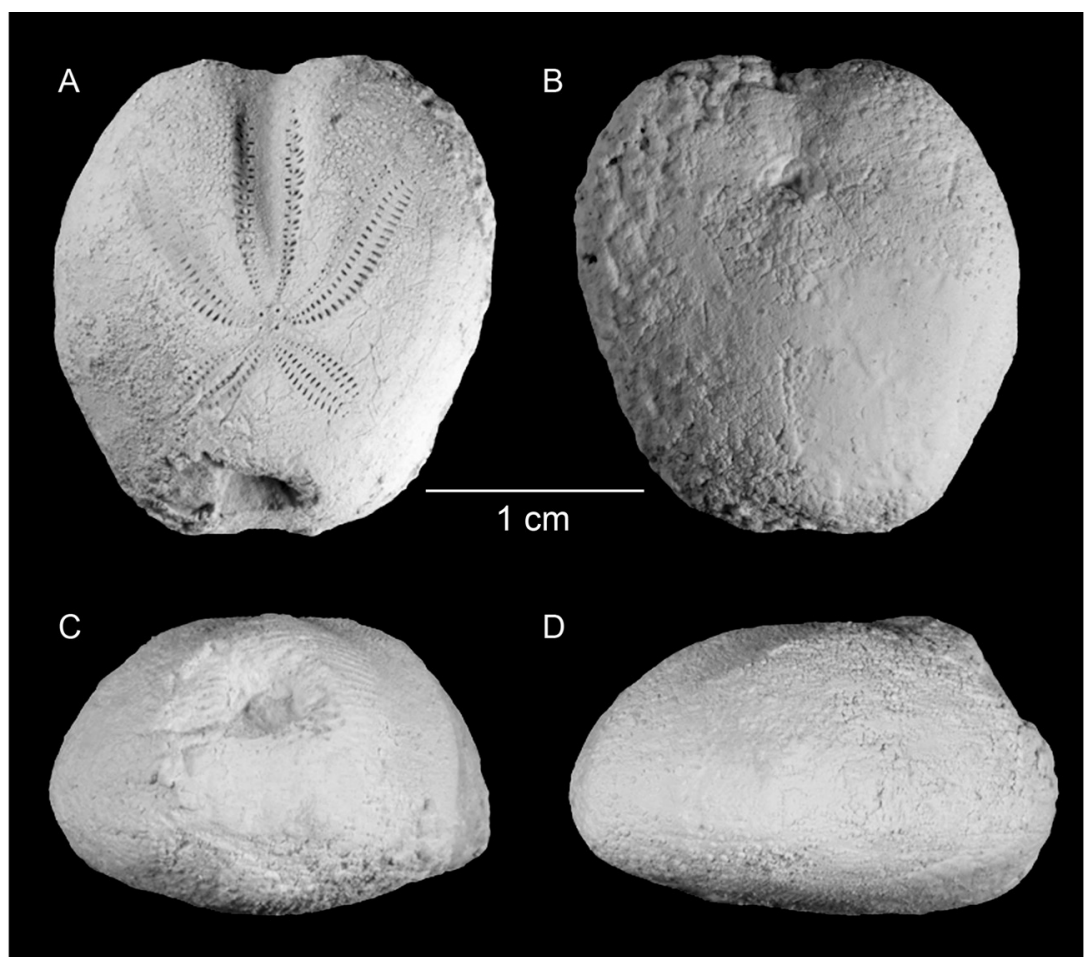

Fig. 11. Washitaster bravoensis (Böse, 1910). FCMP 87 (Albiano). A, vista aboral; B, vista oral; $\mathrm{C}$, vista posterior; $\mathrm{D}$, vista lateral.

Fig. 11. Washitaster bravoensis (Böse, 1910). FCMP 87 (Albian). A, aboral view; B, oral view; $\mathrm{C}$, posterior view; D, lateral view.

Ambulacros anteriores pareados largos, curvos hacia enfrente; ambulacros posteriores pareados aproximadamente tres veces más cortos que los anteriores. Sistema apical central; ápice en el interambulacro posterior. Periprocto pequeño, ovalado y alto, ubicado en la parte alta del borde posterior; peristoma ovalado en posición muy anterior (modificado de Buitrón, 1971).

Distribución. Cretácico; Bell County, Bosque County, Hudspeth County, Williamson County, Presidio County, Grupos Fredericksburg, Washita; Formaciones Comanche Peak, Denison, Duck Creek, Finley, Fort Worth, Georgetown, (Aptiano) Glen Rose, (Cenomaniano) Grayson Marl, Main Street, Wenlock, Texas, E. U. A. Cerro de Muleros y Sierra la Encantada, Chihuahua, México Albiano Superior (Buitrón, 1971).

Material examinado: Sintipos IGM 2274, 2275, 2276, 2277, 2278. Albiano tardío.

\section{Washitaster longisulcus}

(Adkins \& Winton, 1920) Figura 12

Enallaster longisulcus Adkins \& Winton,

1920, p. 55, lám. 9, figs. 4, 8-10. Cooke, 1946, p. 232, lám. 33, figs 5-6.

Washitaster longisulcus. Lambert, 1927,

p. 271. Buitrón, 1971, p.39, lám.12, figs. 1-2, 4 y 6 . Buitrón, 1990, fig. 1-3.

Material tipo. No disponible.

Descripción. Testa ovoidal y angosta, con la superficie oral plana y la superficie aboral convexa. Ambulacro anterior impar abierto, situado en un surco largo y profundo, con pares de poros pequeños, redondos y separados por un gránulo conspicuo. Ambulacros anteriores largos y posteriores muy cortos. Fasciola peripétala múltiple. Sistema apical en posición posterior, con cuatro placas genitales contiguas, las posteriores separadas por la madreporita. 


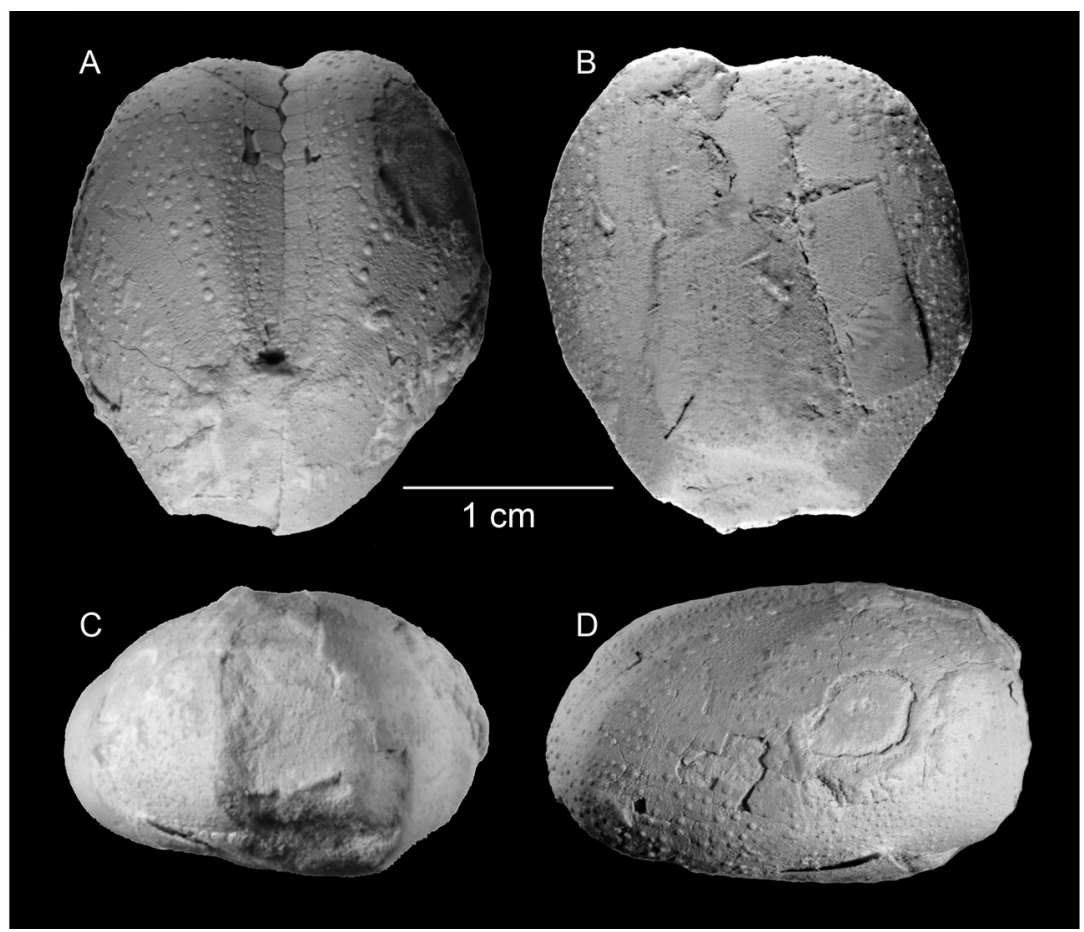

Fig. 12. Washitaster longisulcus (Adkins \& Winton, 1920). IGM 2279 (Cerro de Muleros, Albiano superior). A, vista aboral; B, vista oral; C, vista posterior; D, vista lateral.

Fig. 12. Washitaster longisulcus (Adkins \& Winton, 1920). IGM 2279 (Cerro de Muleros, late Albian). A, aboral view; B, oral view; C, posterior view; D, lateral view.

Periprocto pequeño y ovalado, situado en la superficie posterior de la testa. Peristoma subpentagonal en posición anterior (modificada de Buitrón, 1990).

Distribución. Albiano Superior, Cerro de Muleros, Chihuahua (Buitrón, 1990).

Material examinado: IGM 2279.

\section{FAMILIA Hemiasteridae H. L. Clark, 1917}

Diagnosis. Ambulacros semi-petaloides o rectos, ligeramente curvos; ambulacro impar diferenciado de los ambulacros pares. Fasciola peripétala presente. Sistema apical etmofracto o etmolítico, con cuatro poros genitales, raras veces tres ó dos. Peristoma transverso con labrum desarrollado y placa labral alargada longitudinalmente. Placas episternas biseriadas (Nisiyama, 1968).

Género Hemiaster Desor, 1847
Diagnosis. Testa ancha, más alta que larga, trunca posteriormente. Ambulacro impar no petaloide o semipetaloide con poros redondos y pequeños, ambulacros pares petaloides, bien desarrollados, los anteriores son más largos que los posteriores; con fasciola peripétala. Sistema apical etmofracto o etmolítico, con cuatro poros genitales (modificada de Kier, 1984).

Especie tipo. Spatangus bufo Brongniart, 1822.

Alcance estratigráfico. Cretácico Inferior (Albiano) al Cretácico Superior (Smith y Kroh, 2011).

\section{Hemiaster bexari W.B. Clark, 1915}

Hemiaster bexari W.B. Clark, 1915, p. 89, lám. 46, figs. 1a-e. Sánchez-Rodríguez, 1997, p. 36-37, figs. 16.4-16.6. Stephenson, 1941, p. 65. Akers \& Akers, 1987, p. 104. Hemiaster (Leymeriaster) bexari Adkins, 1928, p.298. 
Material tipo. Holotipo USNM PAL 8330.

Descripción. Testa pequeña y subovalada, más elevada en el margen posterior que en el anterior. Superficie oral plana, con excepción del labrum que es prominente. Superficie aboral cubierta por pequeños tubérculos areolados y con interambulacros poco elevados y superficiales. Ambulacros anteriores mucho más largos que los posteriores, con zonas poríferas anchas, con dos poros transversalmente ovalados. Ambulacro anterior ancho y poco profundo, con poros redondos. Fasciola periambulacral superficial. Sistema apical posterior. El periprocto y se ubica sobre la parte elevada del margen posterior. El peristoma es también pequeño y está localizado cerca del margen anterior (Sánchez-Rodríguez, 1997).

Distribución. Formación Cárdenas, Zamachihue, San Luis Potosí, México. Bexar County y Grupo Navarro, Texas, E.U.A.; Grupo
Difunta, Coahuila (Sánchez-Rodríguez, 1997). La Formación Cárdenas representa un ambiente de laguna cerrada con depósitos de barra, depósito lagunar de baja energía y depósitos de tormenta). Cretácico (Maastrichtiano) (Sánchez-Rodríguez, 1997).

Material examinado: Reporte en Sánchez-Rodríguez, 1997.

\section{Hemiaster calvini W.B. Clark, 1893}

Figura 13

Hemiaster calvini W.B. Clark, 1893, p. 90, figs. 2a-i. Buitrón, 1971, p. 34, lám.10, fig. 2-6. Barrios, 1992, p. 27.

Akers \& Akers, 1987, p. 105.

Material tipo. Sintipo USNM PAL 12241 A y B.

Descripción. Testa de tamaño medio, cordiforme con el borde posterior trunco y casi

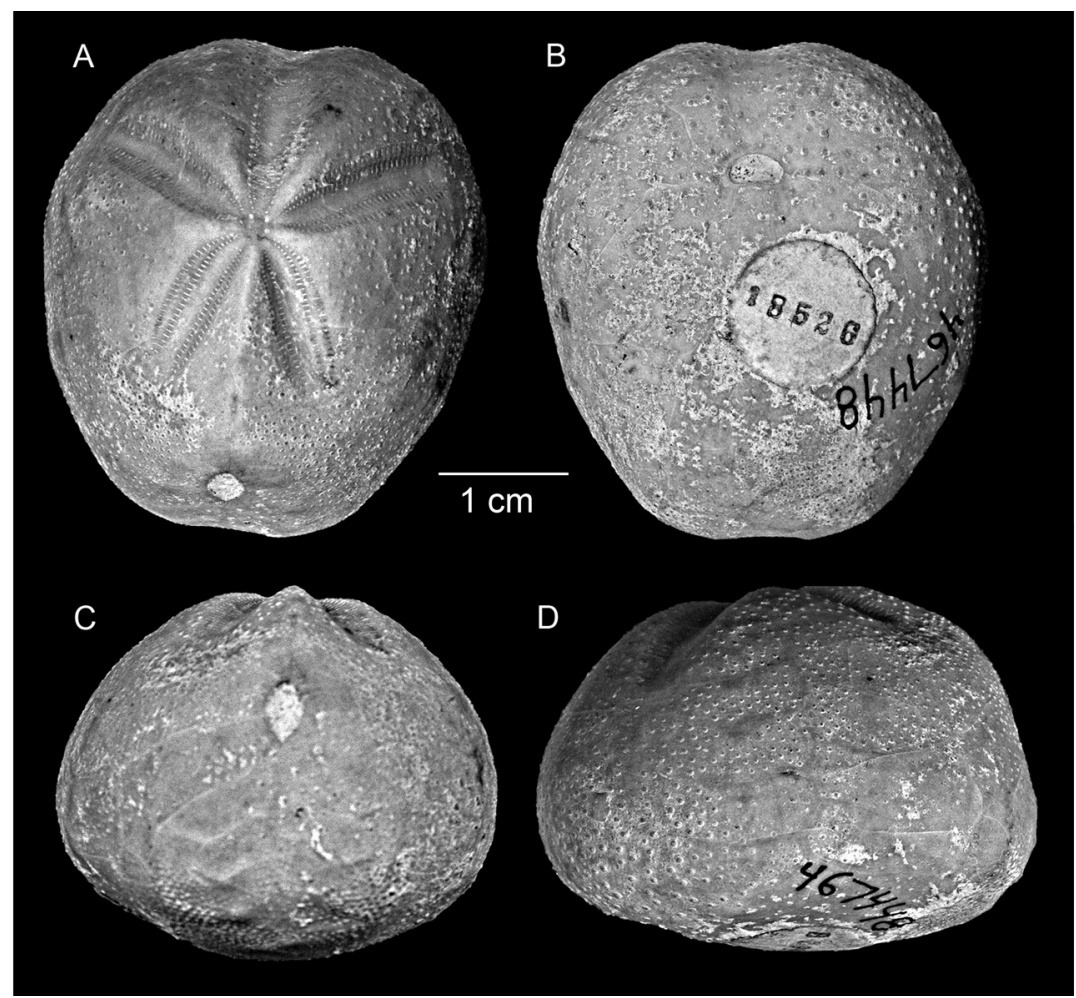

Fig. 13. Hemiaster calvini W.B. Clark, 1893. USNM PAL 467448. A, vista aboral; B, vista oral; C, vista posterior; D, vista lateral.

Fig. 13. Hemiaster calvini W.B. Clark, 1893. USNM PAL 467448. A, aboral view; B, oral view; $\mathrm{C}$, posterior view; D, lateral view. 
vertical; superficie aboral elevada y superficie oral ligeramente convexa. Margen anterior con surco medianamente profundo desde el sistema apical hasta el peristoma. Ambulacro impar en el surco, con zona porífera menos ancha que la interporífera, con poros ovalados y separados por un tubérculo. Ambulacros pares anteriores petaloides largos y anchos, alojados en surcos profundos; la zona porífera tiene poros en forma de ranura del mismo tamaño. Ambulacros posteriores petaloides, cortos y situados en surcos profundos. Área interambulacral posterior elevada; las demás áreas interambulacrales también elevadas, pero en menor grado. Presentan tubérculos pequeños, areolados, crenulados, perforados y numerosas granulaciones más abundantes y más notables en la superficie oral, con fasciola peripétala. Sistema apical ligeramente anterior, con cuatro placas genitales, las posteriores separadas, la placa madrepórica es central y las cinco placas oculares son pequeñas. Periprocto ovalado y situado en la parte posterior trunca. Peristoma pequeño, en la parte anterior de la superficie oral inferior de la testa (Buitrón, 1971).

Distribución. Formaciones Wenlock, Glen Rose (Albiano), Grayson Marl (Cenomaniano), Texas, E. U. A. Cretácico (Albiano superior) en la Formación Aurora, Sierra de Tlahualilo, Coahuila (Buitrón, 1971). Cretácico (Cenomaniano inferior) en la Formación del Río Cerro de Muleros, Chihuahua. Cretácico (Albiano superior) en la Formación Aurora, Sierra de Tlahualilo, Coahuila (Buitrón, 1971). Cretácico (Turoniano) en la Formación Cuautla, al Norte de Cerro de Ahuexotitlán, Chilapa, Guerrero (Barrios, 1992).

Material examinado: USNM PAL 467448.

Hemiaster cholamensis Kew, 1920

Hemiaster cholamensis Kew, 1920,

p. 146-147. Sánchez-Rodríguez, 1997, p. 37, fig. 17.1-17.2.

Material tipo. Museo de Paleontología, Universidad de California en Berkeley, cotipos
UCMP 11338 y 11343 Monterey, California, E. U. A. Cretácico Tardío.

Descripción. Testa pequeña, de ámbito subcircular a subpentagonal; margen posterior ligeramente truncado y más elevado que el anterior, formando una cuña hacia la parte anterior; superficie aboral baja; superficie oral ligeramente convexa. Ambulacros situados en surcos; los pares son delgados y el impar es más ancho y en surco que sigue hasta la superficie oral. Ambulacros posteriores de menos de la mitad del largo que los anteriores, siendo el impar el más largo, con zonas poríferas anchas y con isoporos conjugados. Sistema apical pequeño situado posteriormente al centro de la testa. Periprocto de tamaño medio de forma suboval en posición posterior vertical. Peristoma pequeño, semicircular y con un labrum prominente (modificada de Kew, 1920).

Distribución. Formación Chico, California, E.U.A. (Sánchez-Rodríguez, 1997). Formación Cárdenas, Zamachihue, San Luis Potosí (Sánchez-Rodríguez, 1997). Cretácico (Maastrichtiano), La Formación Cárdenas representa un ambiente de laguna cerrada con aportes de depósito de barra, depósito lagunar de baja energía y depósito de tormenta (Sánchez-Rodríguez, 1997).

Material examinado: Reporte en Sánchez-Rodríguez, 1997.

\section{Hemiaster humphreysanus}

Meek \& Hayden, 1857

Hemiaster humphreysanus

Hayden \& Meek, 1857, p. 47-148.

Cooke, 1942, p.30-31. Sánchez-Rodríguez, 1997, p. 39, fig. 17.3-17.4.

Toxaster (Pliotoxaster) humphreysi

Lambert \& Thiéry, 1924, p. 437.

Material tipo. Tipo USNM PAL 331.

Descripción. Testa de ámbito ovalado; margen posterior trunco. Superficie oral plana, superficie aboral elevada y plana. Ambulacros anchos, profundos y desiguales. Ambulacro impar con pequeños pares de poros separados por un tubérculo pequeño. Ambulacros anteriores largos y los posteriores cortos, 


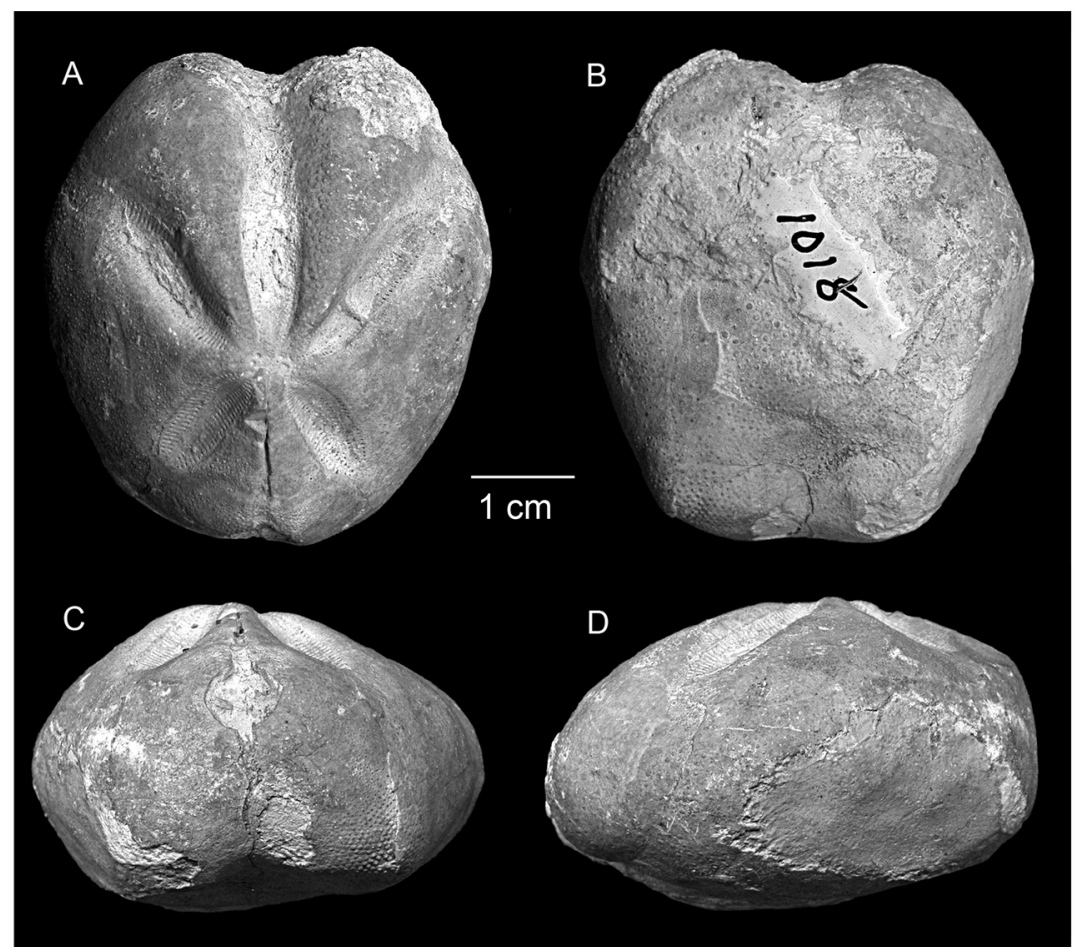

Fig. 14. Hemiaster jacksoni Maury, 1925. Springer Collection, USNM 1018. A, vista aboral; $\mathrm{B}$, vista oral; $\mathrm{C}$, vista posterior; $\mathrm{D}$, vista lateral.

Fig. 14. Hemiaster jacksoni Maury, 1925. Springer Collection, USNM 1018. A, aboral view; $\mathrm{B}$, oral view; $\mathrm{C}$, posterior view; $\mathrm{D}$, lateral view.

de aproximadamente un tercio de la longitud de los anteriores; zonas poríferas amplias, con poros ovalados. Sistema apical posterior. El periprocto es verticalmente ovalado y se halla situado en lo alto de la superficie del margen posterior (Sánchez-Rodríguez, 1997).

Distribución. Lutita Pierre, Wyoming; Formación Mesa Verde, Utah; Arenisca Nacatoch, Arkansas; Formación Ripley, Mississippi, E.U.A. (Sánchez-Rodríguez, 1997). Cretácico (Maastrichtiano) Nuevo León, Formación Potrerillos, y San Luis Potosí, Formación Cárdenas (Sánchez-Rodríguez, 1997).

Material examinado: Reporte en Sánchez-Rodríguez, 1997.

Hemiaster jacksoni Maury, 1925

Figura 14

Hemiaster jacksoni Maury, 1925, p. 519. Buitrón, 1976, p. 22. Akers \& Akers, 1987, p. 106. Sánchez-Rodríguez, 1997,

p. 41-43, fig. 17.5-17.6.

Leiotomaster bösei Smiser, 1936, p. 476,

lám. 64, figs. 4-8.

Hemiaster calvini (Clark). Jones, 1938, p. 135, lám. 13, figs.17-20.

Material tipo. No disponible.

Descripción. Testa de borde subhexagonal, superficie aboral alta, superficie oral plana con el plastrón abultado, superficie posterior vertical. Ambulacro anterior recto y más largo que los ambulacros pares, con poros redondos y pequeños. Ambulacros pares rectos, cerrados en el extremo distal; el par anterior es ligeramente más largo que el par posterior; presentan pares de poros ovalados, más anchos que largos, con zonas poríferas angostas. Fasciola periambulacral angosta y poco visible. Sistema apical tetrabasal, con cuatro placas genitales 
perforadas. Periprocto pequeño, ovalado verticalmente, en la superficie posterior. Peristoma pequeño, en posición anterior y con labrum (modificada de Sánchez-Rodríguez, 1997).

Distribución. Formación Eagle Ford (Turoniano), Terrell County, Texas, E.U.A.

Material examinado: Springer Collection, USNM 1018.

Hemiaster wetherbyi de Loriol, 1887

Figura 15

Hemiaster wetherbyi de Loriol, 1887, p. 391. Cooke, 1953, p. 31-32.

Akers \& Akers, 1987, p. 107.

Hemiaster lacunosus Slocom, 1909, p. 10, lám. 2, figs.2-7.

Hemiaster bexari Clark, 1915, p. 97.

Stephenson, 1941, p. 65.

Hemiaster (Leymeriaster) bexari (Clark).

Lambert \& Thiery, 1924, p. 504.
Hemiaster benhurensis Stephenson 1941, p. 67.

Material tipo. No disponible.

Descripción. Testa pequeña, inflada, con el vértice en su parte posterior; superficie oral convexa; parte posterior trunca. Ambulacros petaloides pares hundidos, rectos y anchos; el par anterior es el doble de largo del par posterior. Ambulacro impar ligeramente hundido y sus poros están separados por un gránulo. Presenta una fasciola peripétala hundida. Sistema apical posterior. Periprocto pequeño y oval, ubicado en la superficie posterior trunca (Akers \& Akers, 1987).

Distribución. Alabama (Maastrichtiano); Formaciones Marlbrook, Nacatoch, Prairie Bluff (Maastrichtiano), Ripley (Maastrichtiano), Saratoga, Arkansas; Pontotoc County

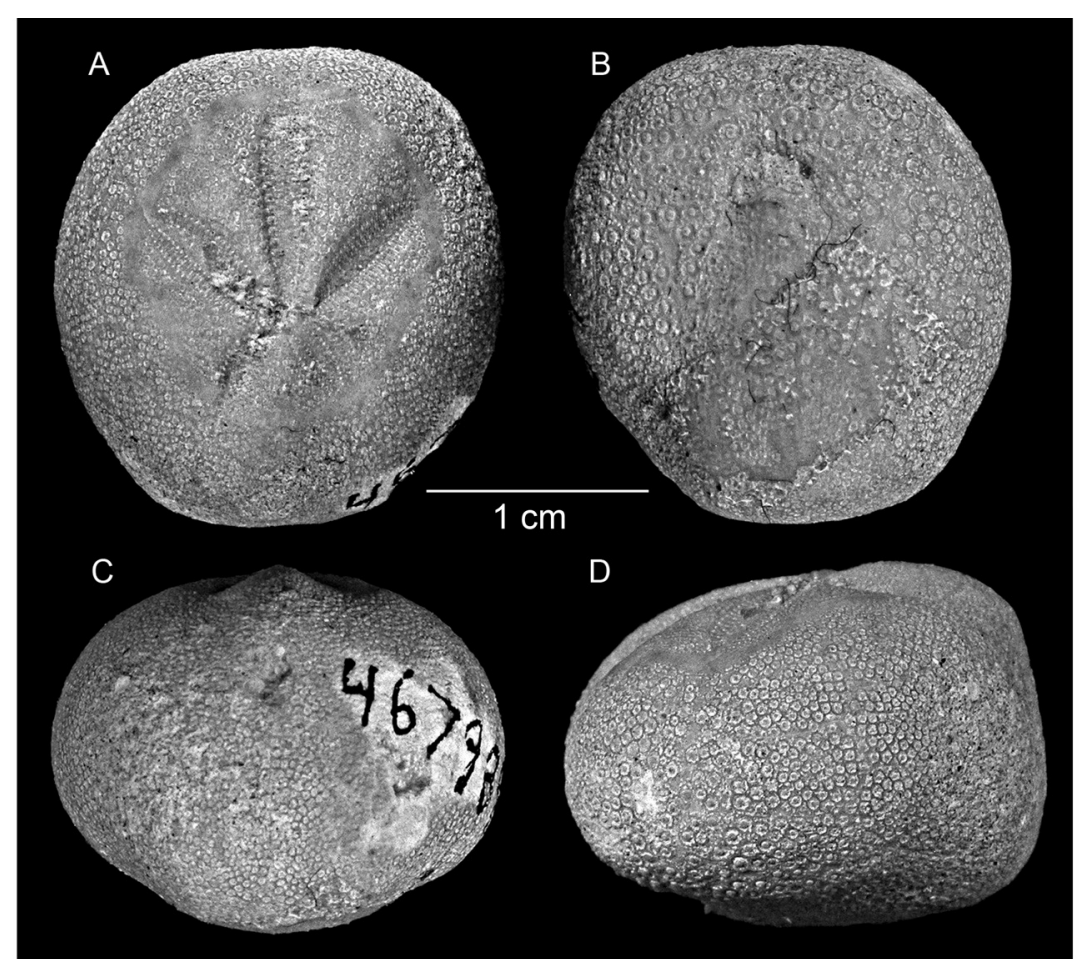

Fig. 15. Hemiaster wetherbyi De Loriol, 1887. USNM PAL 467980. A, vista aboral; B, vista oral; C, vista posterior; D, vista lateral.

Fig. 15. Hemiaster wetherbyi De Loriol, 1887. USNM PAL 467980. A, aboral view; B, oral view; $\mathrm{C}$, posterior view; D, lateral view. 
(Maastrichtiano), Mississippi; Corsicana, Texas, E.U.A. 467980.

\section{Material examinado: USNM PAL}

Hemiaster whitei (W.B. Clark, 1891)

Figura 16

Epiaster whitei W.B. Clark, 1891, p. 77.

Hemiaster whitei W.B. Clark, 1915, p. 77, lám. 43, figs. 2a-c. Buitrón, 1971,

p. 36. lám. 10, figs. 7-9, lám. 11, figs. 1-2.

Akers \& Akers, 1987, p. 108.

Material tipo. Holotipo USNM PAL 9732, Paratipo USNM PAL 4544 y 31202.

Descripción. Testa de contorno cordiforme, más ancha anteriormente y con el borde posterior trunco; superficie aboral ligeramente convexa y superficie oral aplanada; área anterior con un surco profundo desde el sistema apical al peristoma, dividiendo el borde anterior. Ambulacro impar recto, largo, con pares de poros redondos y separados por un tubérculo; ambulacros anteriores largos, continuos hasta el ámbito, ligeramente flexionados y hundidos; con pares de poros en forma de ranuras, más pequeñas en la serie interna. Ambulacros posteriores petaloides muy cortos y abiertos, con pares de poros en forma de ranuras. Fasciola peripétala múltiple. Sistema apical pequeño, excéntrico, anterior, con cuatro placas genitales grandes y la madrepórica central; las oculares no se observan. Periprocto ovalado con los extremos en punta, ubicado en la parte superior del extremo posterior de la testa. Peristoma ligeramente ovalado situado anteriormente (modificada de Buitrón, 1971).

Distribución. Turoniano Sierra de Juárez, Chihuahua (Buitrón, 1971). Formación Cuautla (Turoniano) al Norte de Cerro de Ahuexotitlán, Chilapa, Guerrero (Barrios, 1992).

Material examinado: Springer Collection, USNM.

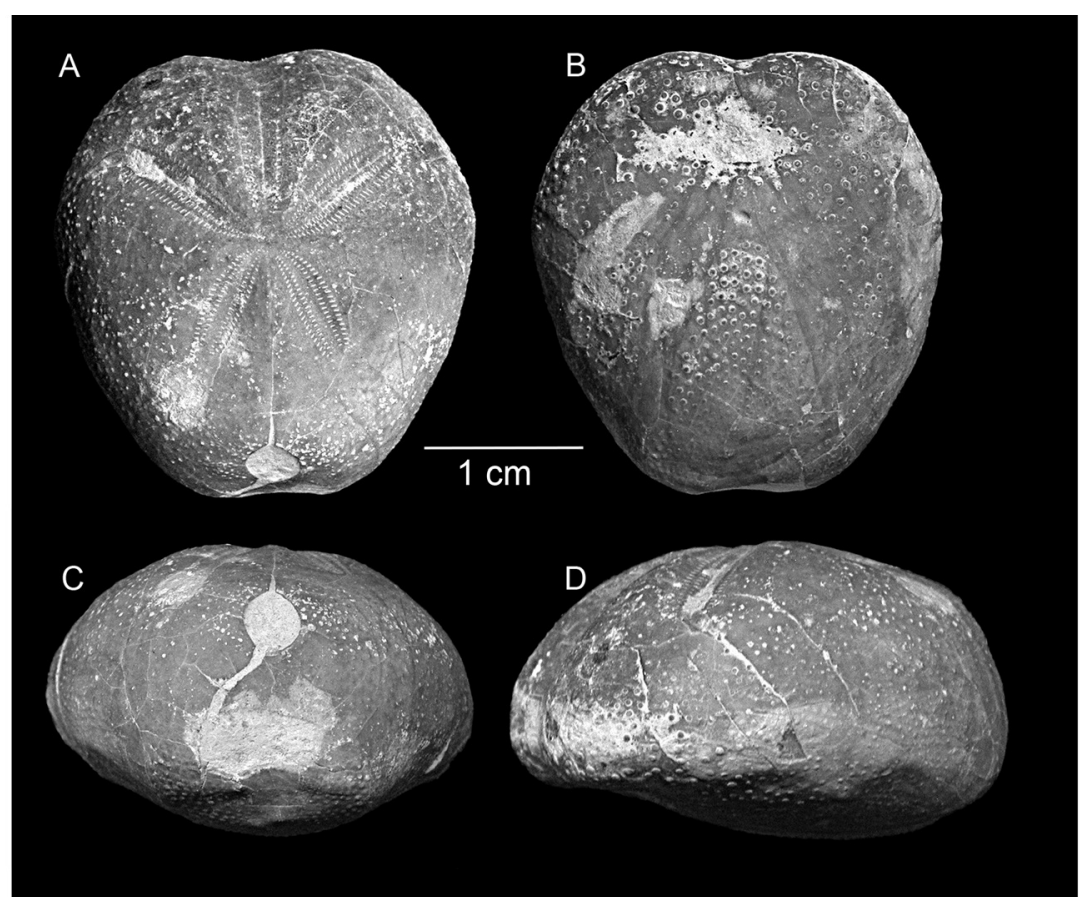

Fig. 16. Hemiaster whitei (W.B. Clark, 1891). Springer Collection, USNM. A, vista aboral; $\mathrm{B}$, vista oral; C, vista posterior; D, vista lateral.

Fig. 16. Hemiaster whitei (W.B. Clark, 1891). Springer Collection, USNM. A, aboral view; $\mathrm{B}$, oral view; $\mathrm{C}$, posterior view; $\mathrm{D}$, lateral view. 
Género Proraster Lambert, 1895

Diagnosis. Testa ovalada, con surco anterior y extremo posterior trunco; de ámbito redondeado. Ambulacro anterior profundo, ancho y con paredes verticales que convergen hacia el ámbito; con pares de poros uniseriales. Ambulacros petaloides hundidos; el par anterior es largo y flexionado; el par posterior de menos de la mitad del largo de los ambulacros anteriores y solo ligeramente hundidos. Fasciola peripétala presente. Sistema apical posterior, etmofracto con 4 poros genitales. Periprocto en la superficie posterior. Peristoma anterior, en contacto con el surco frontal, con labio que cubre al peristoma. Plastrón con placa labial triangular y placas esternales simétricas (Smith \& Kroh, 2011).

Especie tipo. Schizaster atavus Arnaud, 1883.
Alcance estratigráfico. Campaniano a Maastrichtiano (Cretácico) (Smith \& Kroh, 2011).

Proraster dalli (W.B. Clark, 1891)

Figura 17

Hemiaster dalli W.B. Clark, 1891, p. 77, lám. XLVIII, figs. 2a-e.

Hemiaster (Proraster) dalli.

Lambert \& Thiery, 1924, p. 506.

Proraster dalli. Lambert, 1926, p. 273;

Akers \& Akers, 1987, p. 113.

Material tipo. No disponible.

Diagnosis. Testa casi hexagonal, deprimida con pares de poros separados por un puente delgado. Ambulacro impar en un surco ancho y profundo que escota el extremo anterior de la testa. Pares de ambulacros petaloides. Sistema apical posterior. Periprocto ovalado y ubicado

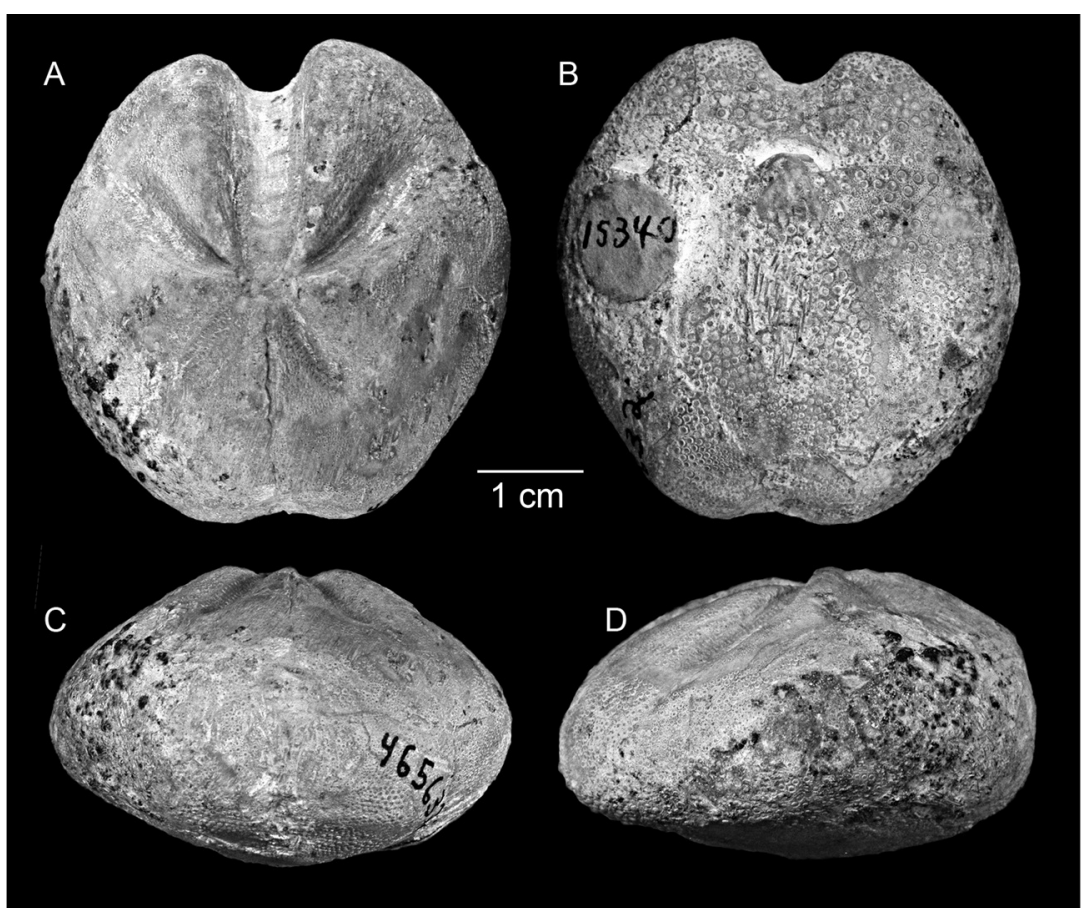

Fig. 17. Proraster dalli (W.B. Clark, 1891). USNM PAL 465632. A, vista aboral; B, vista oral; C, vista posterior; D, vista lateral.

Fig. 17. Proraster dalli (W.B. Clark, 1891). USNM PAL 465632. A, aboral view; B, oral view; $\mathrm{C}$, posterior view; D, lateral view. 
en la superficie posterior, truncada. Peristoma anterior ovalado transversalmente (Akers \& Akers, 1987).

Distribución. Formaciones Anacacho, Corsicana Marl (Maastrichtiano), Texas, E.U.A.

Material examinado: USNM PAL 465632 .

FAMILIA Micrasteridae Lambert, 1920

Diagnosis. Sistema apical etmofracto o transicional con 4 y excepcionalmente con 3 gonoporos. Plastrón mesanfisterno (modificada de López-Jiménez, García-Mataix \& RománLorenzo, 2005).

\section{Género Micraster L. Agassiz, 1836}

Diagnosis. Testa de pequeña a grande, más o menos convexa, de borde cordiforme. Surco anterior variable en profundidad y ancho, visible a nivel del ámbito; con ligeras depresiones laterales. Tubérculos y gránulos miliares pequeños y dispersos que en la superficie oral; grandes y en la superficie aboral compactos. Ambulacros heteromorfos abiertos y variables en profundidad. Ambulacro impar con placas relativamente más anchas y cortas que las del resto de los ambulacros y poros pequeños y redondos separados por un gránulo. Ambulacros pares petaloides, con poros conjugados, los internos redondos y los externos ovalados transversalmente. Sistema apical etmofracto, compuesto de cuatro placas genitales y cinco oculares; oculares I y V haciendo contacto. Superficie posterior amplia, frecuentemente cóncava a la mitad. Fasciola subanal bien desarrollada con pequeños tubérculos, más grandes en las depresiones laterales y más pequeños en el esternón. Peristoma semilunar a ovalado transversalmente. Periprocto redondo a ovalado longitudinalmente con labrum variable en ancho y largo. Interambulacro impar forma un plastrón de tipo anfiesternal (Maczynska, 1968).

Especie tipo. Spatangus coranguinum Leske, 1778.

Alcance estratigráfico. Cretácico Superior (Turoniano) al Paleógeno (Daniano) (Smith \& Kroh, 2011).

Micraster sonorensis Buitrón, 1971

Figura 18

Micraster sonorensis Buitrón, 1971, p. 40-41, lám. 12, fig. 3, 5, 7-9.

Material tipo. Holotipo IGM 2280. Cerro de las Conchas, Arivechi, Sonora (Buitrón, 1971).

Descripción. Testa grande, cordiforme, convexa en el área posterior. Surco poco profundo que escota el margen anterior y se prolonga desde el sistema apical hasta el peristoma. Tubérculos más notables, situados en el plastrón de tipo mesanfisterno, areolados, perforados y crenulados. Ambulacros petaloides, ligeramente hundidos; el anterior corto, del mismo tamaño que los posteriores; la zona porífera es amplia, con poros redondos en la serie interna y ranuras en la serie externa. Ambulacros anteriores largos y rectos, con la zona porífera amplia; posteriores cortos y casi rectos, con una ligera flexión hacia la parte posterior, zona porífera ancha, con poros redondos en la serie interna y con ranuras en la externa. Sistema apical pequeño, anterior, con cuatro placas genitales grandes y cinco oculares triangulares, las posteriores unidas y ligeramente más grandes que las demás. Periprocto redondo, situado en la parte media de la pared posterior, con fasciola subanal (modificado de Buitrón, 1971).

Distribución. Santoniano (Cretácico) en Cerro de las Conchas, Arivechi, Sonora (Buitrón, 1971).

Material examinado: IGM 2280. Cerro de las Conchas, Arivechi, Sonora. 


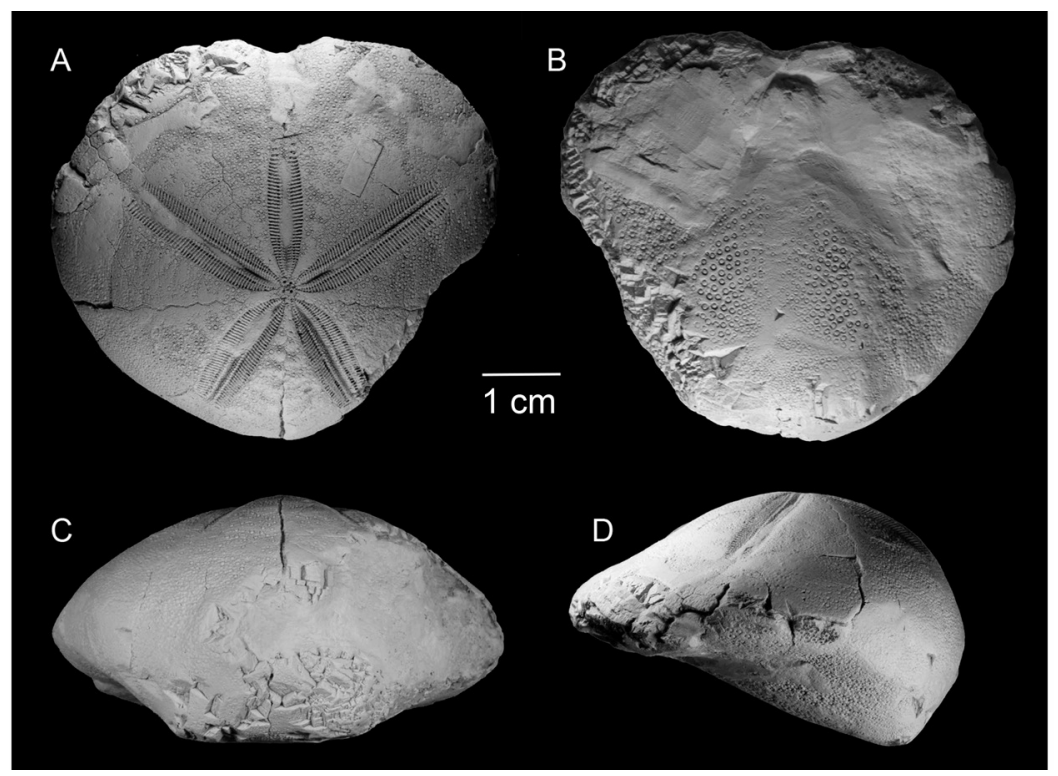

Fig. 18. Micraster sonorensis Buitrón, 1971. Holotipo IGM 2280 (Cerro de las Conchas, Satoniano). A, vista aboral; B, vista oral; C, vista posterior; D, vista lateral.

Fig. 18. Micraster sonorensis Buitrón, 1971. Holotype IGM 2280 (Cerro de las Conchas, Satonian). A, aboral view; B, oral view; C, posterior view; D, lateral view.

\section{AGRADECIMIENTOS}

Martínez Melo agradece al CONACYT la beca de doctorado No. 35167. A Ma. del Carmen Perrillat y Jesús Alvarado encargados de la Colección Nacional de Paleontología, IGUNAM, el haber proporcionado material para su estudio. A Francisco Sour (FCMP-UNAM) y Dan Levin (Smithsonian Museum of Natural History) por su apoyo para la revisión de los ejemplares en las diferentes colecciones. A Josep Anton Moreno Bedmar por el apoyo en la fotografía de los ejemplares. A Greta Ramírez y a Saraí Hernández por su colaboración en la revisión del escrito.

\section{RESUMEN}

En México, los equinoideos constituyen una parte muy importante de la fauna fósil, tanto del Mesozoico como del Cenozoico, que es poco conocida. Las especies de espatangoides se encuentran ampliamente representadas en varios afloramientos fosilíferos del Cretácico, los cuales tienen alto valor bioestratigráfico y paleoecológico. La revisión de los equinoideos Cretácicos del orden Spatangoida L. Agassiz, 1840 de México, permitió actualizar la taxonomía de las especies y contribuyó al estudio de su distribución paleogeográfica y estratigráfica. El orden Spatangoida es el más diverso en México con tres familias (Toxasteridae, Hemiasteridae, Micrasteridae), siete géneros (Epiaster, Heteraster, Macraster, Washitaster, Hemiaster, Proraster, Micraster) y 21 especies. La familia mejor representada es Toxasteridae con el $54 \%$ de las especies reportadas para el Cretácico de México. Los espatangoides del Cretácico de México se encuentran en diferentes localidades cretácicas en los estados de Sonora (Cerro de las Conchas), Chihuahua (Cerro de Muleros, Sierra de Juárez, Placer de Guadalupe, Sierras La Ranchera, El Presidio, Sierra Banco de Lucero, Sierra La Encantada, Villa Ahumada), Coahuila (Sierra de Tlahualilo), Nuevo León (Sinclinal de Delgado), San Luis Potosí (Zamachihue), Colima (E1 Rosario), Puebla (San Juan Raya, Barranca Salitrillo), Guerrero (Cerro de Ahuexotitlán) y Oaxaca (Las Huertas).

Palabras clave: Equinoideos Irregulares; Spatangoida; Cretácico; México.

\section{REFERENCIAS}

Akers, R.E. \& Akers, T.J. (1987). Texas Cretaceous echinoids. Texas Paleontology Series Publication, 3, 1-143.

Adkins, W.S. (1920). The Weno and Pawpaw formations of the Texas Comanchean. University of Texas Bulletin, 1(2), 1-257. 
Adkins, W.S. (1928). Handbook of Texas Cretaceous fossils. Texas, E.U.A.:University of Texas at Austin.

Adkins, W.S. \& Winton, W.M. (1920). Paleontological correlation of the Fredericksburg and Washita formations in North Texas. University of Texas Bulletin, $1945,1-128$.

Barrios, S.(1992).Equinoideos(Echinodermata:Echinoidea) del Cretácico Superior de Ahuexotitlán, Guerrero; implicaciones paleogeográficas y paleoecológicas (Tesis de licenciatura). Escuela de Ciencias Químico-Biológicas, Universidad Autónoma de Guerrero, Guerrero, México.

Böse, E. (1910). Monografía geológica y paleontológica del Cerro de Muleros cerca de Ciudad Juárez y descripción de la fauna cretácea de la Encantada, Placer de Guadalupe, Estado de Chihuahua. Boletín del Instituto de Geología, Mexico, 25, 1-193.

Böse, E. \& Calvins, O.A. (1927). The Cretaceous and Tertiary of Southern Texas and Northern Mexico. University of Texas Bulletin, 2748, 7-142.

Buitrón, B.E. (1968). Catálogo de Equinoides fósiles de México. Paleontología Mexicana, 26, 1-50.

Buitrón, B.E. (1970). Equinoides del Cretácico Inferior de la Región de San Juan Raya-Zapotitlán, estado de Puebla. Paleontología Mexicana, 30, 1-46.

Buitrón, B.E. (1971). Equinoides del Cretácico del Norte de México. Paleontología Mexicana, 31, 1-70.

Buitrón, B.E. (1976). Tres especies de equinoides (Echinodermata: Echinoidea) del Cretácico Superior de Atenango del Río, Guerrero. Boletín de la Sociedad Geológica Mexicana, 37, 18-24.

Buitrón, B.E. (1978). Distribución de los equinoides terciarios en la planicie costera del Golfo de México, en América Central, en el Norte de América del Sur y en las Antillas, Instituto de Geología, Boletín, $101,66-114$

Buitrón, B.E. (1990). Catálogo de Equinoides del Jurásico Superior y del Cretácico Inferior de México. Instituto Mexicano del Petróleo.

Buitrón, B.E. \& Solís-Marín, F.A. (1993). La biodiversidad en los equinodermos fósiles y recientes de México. Revista Sociedad Mexicana de Historia Natural, Vol. Esp. 44, 209-231.

Clark, H.L. (1917). The Echinoneidae, Nucleolitidae, Urehinidae, Echinocorythidae, Calymnidae, Porurtalesiidae, Paleostomatidae, Aeropsidae, Palaeopneustidae, Hemiasteridae and Spatangidae. Memoirs of the Museum of Comparative Zoology at Harvard College, 46(12), 85-283.
Clark, W.B. (1891). A revision of the Cretaceous Echinoidea of North America. Johns Hopkins University Circulars, 10(89), 75-77.

Clark, W.B. (1893). The Mesozoic Echinodermata of the United States. United States Geological Survey Bulletin, 97, 1-207.

Clark, W.B. (1915). The Mesozoic Echinodermata. United States Geological Survey Bulletin, 54(1), 9-100.

Cooke, C.W. (1942). Cenozoic irregular echinoids of eastern United States. Journal of Paleontology, 16(1), 1-62.

Cooke, C.W. (1946). Comanche echinoids. Journal of Paleontology, 20(3), 193-237.

Cooke, C.W. (1953). American Upper Cretaceous Echinoidea. A Shorter Contribution to General Geology. Geological Survey Professional Paper, 254-A, 1-44.

Cooke, C.W. (1955). Some Cretaceous echinoids from the Americas. A Shorter Contribution to General Geology, Geological Survey Professional Paper, 264-E, 87-112.

Cotteau, G. (1890). Échinides eocènesde la province d'Alicante. Mémoires de la Société Géologique de France, 3(5), 1-72.

De Loriol, P. (1887). Notes pour servir a l'etude des échinodermes. Recueil Zoologique, Suise, 4, 365-407.

Finsley, Ch.E. (1999). A field guide to fossils of Texas. Maryland, E.U.A.: Gulf Publishing.

Fischer, A.G. (1966). Spatangoids. En R.C. Moore, (Ed.). Treatise on Invertebrate Paleontology, part U. Echinodermata 3. Kansas, E.U.A.: The Geological Society of America and The University of Kansas Press.

Jones, T.S. (1938). Geology of the Sierra de la Peña and Paleontology of the Indidura Formation, Coahuila, México. Geological Society of America Bulletin, 49, 69-149.

Kew, W. (1920). Cretaceous and Cenozoic Echinoidea of the Pacific Coast of North America. Bulletin of the Department of Geology, University of California Publication, 12(1), 23-236.

Kier, P. (1984). Fossil spatangoid echinoids of Cuba. Washington D.C., E.U.A.: Series Publications of the Smithsonian Institution.

Kroh, A. \& Smith, A.B. (2010). The phylogeny and classification of post-Paleozoic echinoids. Journal of Systematic Palaeontology, 8(2), 147-212.

Kühn, O. (1925). Die echinodermen der Gosauformation. Annalen des Naturhistorischen Museum im Wein, 39, 177-189. 
Lambert, J. (1927). Notes sur Quelques Echinides recueillis par Mr. Walther Staub dans le Neogene de l'est du Mexique. Eclogae Geologica Helvetia, 21(1), 272-283.

Lambert, J. \& Thiéry, P. (1924). Essai de nomenclature raisonnée des Échinides.

López-Jiménez, J., García-Mataix, J.L., \& Román-Lorenzo E. (2005). Equinoideos fósiles de la provincia de Albacete. SABUCO, Revista de estudios albacetenses, 5, 179-246.

Mączyńska, S.S. (1968). Echinoids of the genus Micraster L. Agassiz from the Upper Cretaceous of the CracowMiechów area. Prace Muzeum Ziemi, 12, 87-168.

Maury, C.J. (1925). Fosseis terciarios do Brazil com descripcao de novas formas cretaceas. Monographias do Servicio Geologico e Mineralogico do Brazil, 4, 1-75.

Hayden, F. V. \& Meek, F. B. (1857). Descriptions of New Species and Genera of Fossils, Collected by Dr. FV Hayden in Nebraska Territory, under the Direction of Lieut. GK Warren, US Topographical Engineer; with Some Remarks on the Tertiary and Cretaceous Formations of the North-West, and the Parallelism of the Latter with Those of Other Portions of the United States and Territories. Proceedings of the Academy of Natural Sciences of Philadelphia, 9, 117-133.

Nieto, I. \& García, P. (2006). Cretaceous echinoids of México. En Vega, F.J., T.G. Nyborg, M.C. Perrilliat, M. Montellano-Ballesteros, S.R.S. Cevallos-Ferriz y S.A. Quiroz-Barroso. Studies on Mexican Paleontology. México, México: Springer.
Nisiyama, S. (1968). The echinoid fauna from Japan and adjacent regions. Tokyo, Japón: Geological Institute, Faculty of Science, University of Tokyo.

D’Orbigny, A. (1853). Échinoides irréguliers. Paléontologie francaise, Terrain crétacé, series, 1(6), 1-596.

Prothero, D.R. \& Dott, R.H. (2004). Evolution of the Earth, $7^{a}$ Edición. New York, E.U.A.: Mc Graw Hill, Higher Education.

Sánchez-Rodríguez, M.A. (1997). Paleobiogeografía de equinoideos del Cretácico Superior (Maastrichtiano) de Nuevo León, San Luis Potosí y Guerrero. Tesis de Licenciatura. Facultad de Ciencias, UNAM. 88p.

Slocom, A.W. (1909). New echinoids from the Ripley Group of Mississippi. Field Museum of Natural History, 4(1), 1-16.

Smiser, J.S. (1936). Cretaceous echinoids from trans-Pecos Texas. Journal of Paleontology, 10(6), 449-480.

Smith, A.B. (1984). Echinoid Palaeobiology. Londres, Inglaterra: George Allen \& Unwin.

Smith, A.B. \& Kroh, A. (2011). The Echinoid Directory. World Wide Web electronic publication.http://www. nhm.ac.uk/research-curation/projects/echinoid-directory/index. Abril 20, 2017.

Stephenson, L.W. (1941). The larger invertebrate fossils of the Navarro Group of Texas. University of Texas Bulletin, 4101(1), 1-641.

Whitney, F.L. (1916). The Echinoidea of the Buda Limestone. Bulletins of American Paleontology, 26, 1-36. 